\title{
Using the Richmond Fed Manufacturing Survey to Gauge National and Regional Economic Conditions
}

Nika Lazaryan and Santiago M. Pinto

$\mathrm{S}$ everal Regional Banks in the Federal Reserve System conduct regional surveys of business conditions in an effort to obtain realtime information about changes in local economic conditions. To the extent that the performance of the national economy is related to the performance of its regions, the regional surveys may provide useful information about national economic conditions as well. The results of the monthly regional surveys receive attention among analysts and other organizations that assess and forecast economic conditions because they are typically available one or two weeks prior to the release of the national and regional data. Considering how the survey data are used, it is extremely important, first, to understand what the surveys actually measure and, second, to determine how well they measure changes in economic conditions. This paper intends to offer some insights on these issues by carefully analyzing the underlying survey data and investigating their ability to precisely gauge economic conditions observed at both national and regional levels.

The present work focuses on the information content of the Regional Surveys of Business Activity conducted by the Federal Reserve Bank of Richmond (FRBR). We specifically examine the survey that

Federal Reserve Bank of Richmond: nika.lazaryan@rich.frb.org; Federal Reserve Bank of Richmond: santiago.pinto@rich.frb.org. The views expressed in this paper are those of the authors and should not necessarily be interpreted as those of the Federal Reserve Bank of Richmond or the Federal Reserve System.

DOI: https://doi.org/10.21144/eq1030103 
tracks the manufacturing sector, the Fifth District Survey of Manufacturing Activity. In general, the surveys collect qualitative data from businesses in the Fifth District on several items that are supposed to convey information about recent changes in economic activity. For instance, survey participants are asked if they have observed an increase, a decrease, or no change in their levels of employment, shipments, orders, and wages, as well as other indicators. The responses are summarized into a statistic, called a diffusion index, that essentially captures the breadth of the changes taking place along the relevant economic dimensions in the time period under consideration. A few individual diffusion indices are combined into a composite diffusion index. In this paper, we evaluate the performance of these individual and composite diffusion indices by examining how closely they track overall national and regional economic conditions.

As a measure of national economic activity, this paper uses the manufacturing diffusion index produced by the Institute of Supply Management (ISM). The individual diffusion indices calculated by the ISM are based on questions that are very similar to those included in the FRBR survey. Previous work, such as Harris et al. (2004), shows that the composite ISM index is a good gauge of national economic activity based on its ability to track the national gross domestic product (GDP) and personal income. Their work also shows that there is indeed a strong correlation between the ISM and the indices produced from regional surveys by the Richmond and Philadelphia Federal Reserve Banks. ${ }^{1}$

Our indicator of regional economic activity in the Fifth District is a weighted average of state payroll manufacturing employment growth (MEG) rates. Only a few papers have attempted to evaluate the accuracy of the diffusion indices produced by regional Reserve Banks in describing economic changes at the regional or local level. The limited work in this area includes Harris et al. (2004) and Pinto et al. (2015b). Even though the analysis in Harris et al. (2004) focuses on the national economy, it also briefly assesses the extent to which the FRBR composite diffusion index helps explain changes in personal consumption expenditures (PCE) in the Fifth District. We use MEG instead of PCE because, among other reasons, the former series are available monthly, whereas the latter are available only at quarterly intervals. Our work is also related to Pinto et al. (2015a). This paper assesses the ability of certain specific individual diffusion indices (employment and wage diffusion indices) to explain employment and wage growth rates. It argues that, in general, growth rates include information both about

\footnotetext{
${ }^{1}$ It would be useful, in future analysis, to benchmark the FRBR indices against other measures of economic activity, such as the Industrial Production Index.
} 
the "intensive margin," or the size or magnitude of a change, and the "extensive margin," or the spread or breadth of a change. As a result, diffusion indices would describe fairly well how a variable changes over time, if those changes are predominantly explained by a higher proportion of participants reporting a change (up, down, or remain the same). Among other things, this paper shows that while the FRBR employment diffusion index tends to track quite well regional employment growth, it does not do a good job at tracking wage growth.

The analysis performed in this paper departs from the previous work in at least two fundamental ways. First, we carefully examine the behavior of all diffusion indices currently reported by the FRBR, with the intention of gaining a much broader understanding of the information conveyed by the FRBR survey. The conclusions of this study may be used to verify whether the FRBR indices capture what they intend to capture and to determine which indices are more informative depending on the specific objective. Second, this exercise offers useful insights that could guide the design and construction of alternative indicators of economic activity and provide feedback on what kind of data to gather (for instance, which survey questions are more relevant, or how representative the survey sample is).

Our analysis proceeds as follows. We first evaluate how well the FRBR composite diffusion index tracks national and regional economic activity, as measured by the ISM diffusion index and the Fifth District MEG, respectively. We next explore the differential contribution of the individual components of the composite diffusion index. Finally, we explore the benefits of including information that is currently available from the FRBR surveys but is not considered in the calculation of the composite index. Our findings show, among other things, that while the reported composite index contains useful information about economic activity at both the national and regional levels, models that incorporate additional survey information may improve the predictive power of the FRBR indices.

The rest of this paper is organized as follows. Section 1 describes the survey and the data used in the analysis. Section 2 evaluates the ability of FRBR diffusion indices to describe the behavior of the national economy. Section 3 examines the relationship between the FRBR diffusion indices and economic conditions in the Fifth District. Section 4 summarizes and discusses the results.

\section{PRELIMINARY ANALYSIS OF THE DATA}

The work in this paper focuses on the Fifth Federal Reserve District, which includes Virginia, most of West Virginia, Maryland, North 
Carolina, South Carolina, and the District of Columbia. Moreover, the analysis is centered on the manufacturing sector. Three pieces of data are used: the diffusion indices produced by the FRBR, constructed from the information collected by the Fifth District Survey of Manufacturing Activity; the manufacturing diffusion indices reported by the ISM, used as a proxy of national economic activity in the manufacturing sector; and Fifth District payroll MEG, used as a measure of regional manufacturing activity. The data are monthly, and the sample covers the period from May 2002 to June 2017.

The FRBR conducts monthly surveys within the Fifth Federal Reserve District states to assess business conditions in two sectors: manufacturing and service. The manufacturing survey is designed to approximate the distribution of manufacturing firms by state, industry type, and firm size. ${ }^{2}$ It inquires about various aspects related to the economic conditions faced by firms, including questions on employment, shipments, new orders, backlogs, inventories, prices, etc. ${ }^{3}$ The survey is qualitative in nature, in the sense that firms are asked whether they experienced an increase, decrease, or no change in each variable of interest from the preceding month. The responses to each question are then combined into what is referred to as a diffusion index. ${ }^{4}$

The diffusion index calculated by the FRBR is similar to the manufacturing diffusion index reported by the ISM. The latter, however, is based on information collected through a survey of more than 300 purchasing managers of manufacturing companies across the US. This survey is nationally representative and captures the various manufacturing categories by their relative contribution to the GDP.

In general, diffusion indices are summary statistics of the form

$$
D_{t}=100 \times\left(w^{u} \frac{N_{t}^{u}}{N_{t}}+w^{s} \frac{N_{t}^{s}}{N_{t}}+w^{d} \frac{N_{t}^{d}}{N_{t}}\right),
$$

where $N_{t}^{u}, N_{t}^{s}$, and $N_{t}^{d}$ denote, respectively, the number of survey participants who report that the relevant economic variable has increased, stayed unchanged, or declined from period $(t-1)$ to period $t$. The

\footnotetext{
${ }^{2}$ On average, and during the sample period under consideration, the number of respondents in the manufacturing survey has oscillated around 100.

${ }^{3}$ The survey also includes questions on vendors, average workweek hours, wages, business expenditures, inventories of raw materials and finished goods, as well as capacity utilization.

${ }^{4}$ The FRBR currently reports diffusion indices at the Fifth District level. Sample sizes are at the moment too small to report informative diffusion indices at the state level. In this paper, the focus is precisely on the regional diffusion index because, as explained earlier, one of the goals is to determine whether information about the economic performance of the region conveys useful information about the national economy. See Pinto et al. (2015b) for a thorough discussion about the information content of statelevel diffusion indices.
} 
FRBR, for example, reports diffusion indices with $w^{u}=1, w^{s}=0$, and $w^{d}=-1$, so the range of the index is $[-100,100]$. Note that, in this case, the diffusion index is simply the difference between the fraction of respondents who reported an increase and the number of respondents who reported a decrease in a particular measure of economic activity. Therefore, a positive (negative) reading indicates that the proportion of participants who report an increase is higher (lower) than the proportion of those who report a decline in the variable. A larger value of the index (in absolute terms) indicates that the change in the economic variable is widely spread out and broadly observed among respondents. ${ }^{5}$ The diffusion indices reported by the ISM use $w^{u}=1, w^{s}=0.5$, and $w^{d}=0$. The range of this index is $[0,100]$, so in this instance, the series are centered at 50: a reading of the index above (below) 50 indicates that the percentage of responses reporting an increase is higher (lower) than the percentage of responses indicating a decline. Both the FRBR and ISM also report composite indices that consist of a weighted average of several individual diffusion indices, each one tracking different indicators of economic activity. Specifically, the composite index reported by the FRBR is given by

$$
R I C_{t}=0.27 \times R I C_{t}^{E}+0.33 \times R I C_{t}^{S}+0.40 \times R I C_{t}^{O},
$$

and the ISM composite index by

$$
I S M_{t}=0.20 \times\left(I S M_{t}^{E}+I S M_{t}^{S}+I S M_{t}^{O}+I S M_{t}^{P}+I S M_{t}^{I}\right),
$$

where $R I C_{t}^{i}$ and $I S M_{t}^{i}$ are the FRBR and ISM individual diffusion indices for category $i$, and the superscript $i$ stands for $E$ : employment, $S$ : shipments, $O$ : orders, $P$ : production, and $I$ : inventories. ${ }^{6}$ When comparing the FRBR diffusion index to the ISM, we normalize the FRBR diffusion index in order to compare both indices on the same scale. Finally, to measure regional economic activity in the manufacturing sector, we use the series of payroll MEG obtained from the Bureau of Labor Statistics (BLS). We calculate the Fifth District MEG as a weighted average of the states' MEG. $^{7}$ For the purposes of our analysis, the closest counterpart to the manufacturing FRBR diffusion indices is regional MEG, not only because MEG closely tracks changes

\footnotetext{
${ }^{5}$ See Pinto et al. (2015a) or Pinto et al. (2015b) for a thorough explanation of diffusion indices.

${ }^{6}$ The weights currently used in the FRBR composite index $R I C_{t}$ were obtained from Harris et al. (2004). Note, however, that the weights in that paper were chosen with the explicit goal of comparing the ISM and FRBR diffusion index series. In other words, the FRBR composite index, with those specific weights, was intended to track changes at the national level.

${ }^{7}$ We apply our own seasonal adjustment process to the ISM, MEG, and FRBR diffusion index series to preserve uniformity.
} 
Table 1 Summary Statistics

\begin{tabular}{lllll}
\hline & & & & \\
& Variable & Mean & Std. Dev. & N \\
$I S M_{t}$ & Composite ISM & 52.765 & 4.810 & 182 \\
$M E G_{t}$ & Manufacturing employment growth & -0.172 & 0.386 & 181 \\
$R I C_{t}$ & FRBR manu. composite diffusion index & 50.366 & 5.790 & 182 \\
$R I C_{t}^{S}$ & Shipments & 50.820 & 6.462 & 182 \\
$R I C_{t}^{O}$ & Orders & 50.363 & 6.904 & 182 \\
$R I C_{t}^{E}$ & Employment & 49.817 & 4.980 & 182 \\
$R I C_{t}^{B}$ & Backlog & 45.410 & 5.667 & 182 \\
$R I C_{t}^{C}$ & Capacity & 49.304 & 5.847 & 182 \\
$R I C_{t}^{V}$ & Vendors & 52.032 & 3.354 & 182 \\
$R I C_{t}^{H}$ & Hours & 49.531 & 4.978 & 182 \\
$R I C_{t}^{W}$ & Wages & 54.826 & 2.828 & 182 \\
$R I C_{t}^{I F}$ & Inventory finished goods & 59.627 & 4.036 & 182 \\
$R I C_{t}^{I R}$ & Inventory raw materials & 58.006 & 3.422 & 182 \\
\hline
\end{tabular}

in the manufacturing sector, but also because the data are available at monthly intervals. ${ }^{8}$

\section{Descriptive analysis of the series}

We start our examination with a simple descriptive analysis of the series. Consider first the $R I C_{t}$ and $I S M_{t}$ series. The summary statistics reported in Table 1 indicate that, for the period under consideration, the average value of $I S M_{t}$ is higher than the average of $R I C_{t}$, but the volatility of $R I C_{t}$ is more pronounced. ${ }^{9}$ Moreover, from Figures 1a and $1 b$, it appears that the series follow each other very closely.

Figure 2a describes the behavior of $M E G_{t}$ (measured on the left axis) and $R I C_{t}$ (measured on the right axis), and Figure 2b shows a scatter plot of the two series. ${ }^{10}$ It appears from the two figures that the FRBR composite diffusion index tracks fairly well the Fifth District MEG. In Pinto et al. (2015b), we find that the FRBR employment diffusion index also follows very closely the behavior of regional employment growth. In Section 3, we will evaluate in more detail the differential contribution of each one of the FRBR diffusion index series in explaining regional economic changes.

\footnotetext{
${ }^{8}$ When comparing the regional MEG and the FRBR diffusion indices, we use the currently reported version of the FRBR diffusion index with $w^{u}=1, w^{s}=0$, and $w^{d}=$ -1 .

${ }^{9}$ The higher volatility of $R I C_{t}$ may be partly attributed to a smaller sample size.

${ }^{10}$ In Figures $2 \mathrm{a}$ and $2 \mathrm{~b}$, we use the FRBR diffusion index series centered at zero.
} 
Figure $1 I S M_{t}$ and $R I C_{t}$

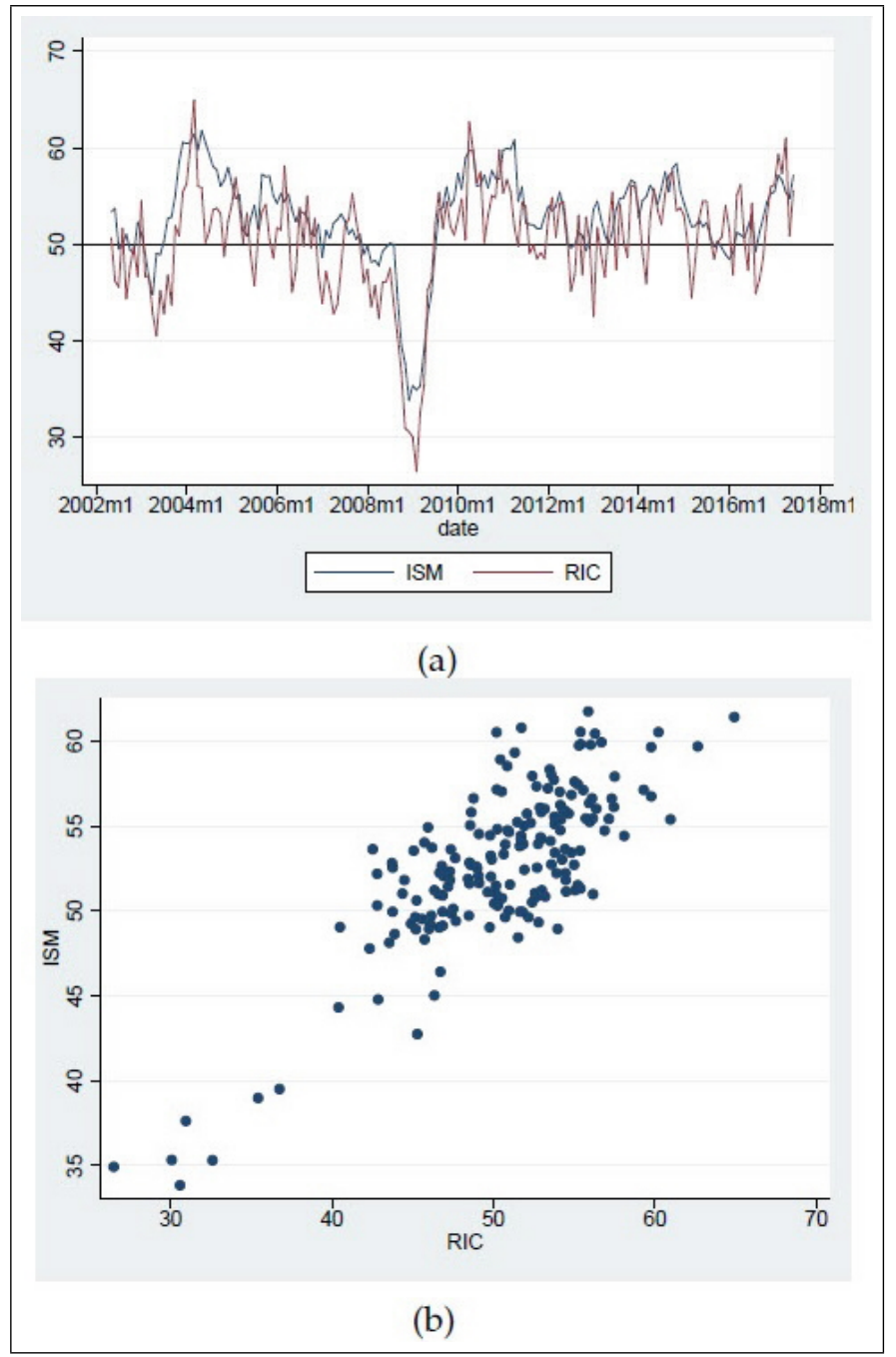

Figure 3a shows the evolution of the individual components of the composite index $R I C_{t}$ : $R I C_{t}^{E}, R I C_{t}^{S}$, and $R I C_{t}^{O}$. While the $R I C_{t}^{O}$ series shows the largest volatility, the $R I C_{t}^{E}$ series shows the least. Additional diffusion indices obtained from other questions in the FRBR survey are shown in Figure 3b. Among all the series, $R I C_{t}^{W}$ has the lowest standard deviation. 
Figure $2 M E G_{t}$ and $R I C_{t}$

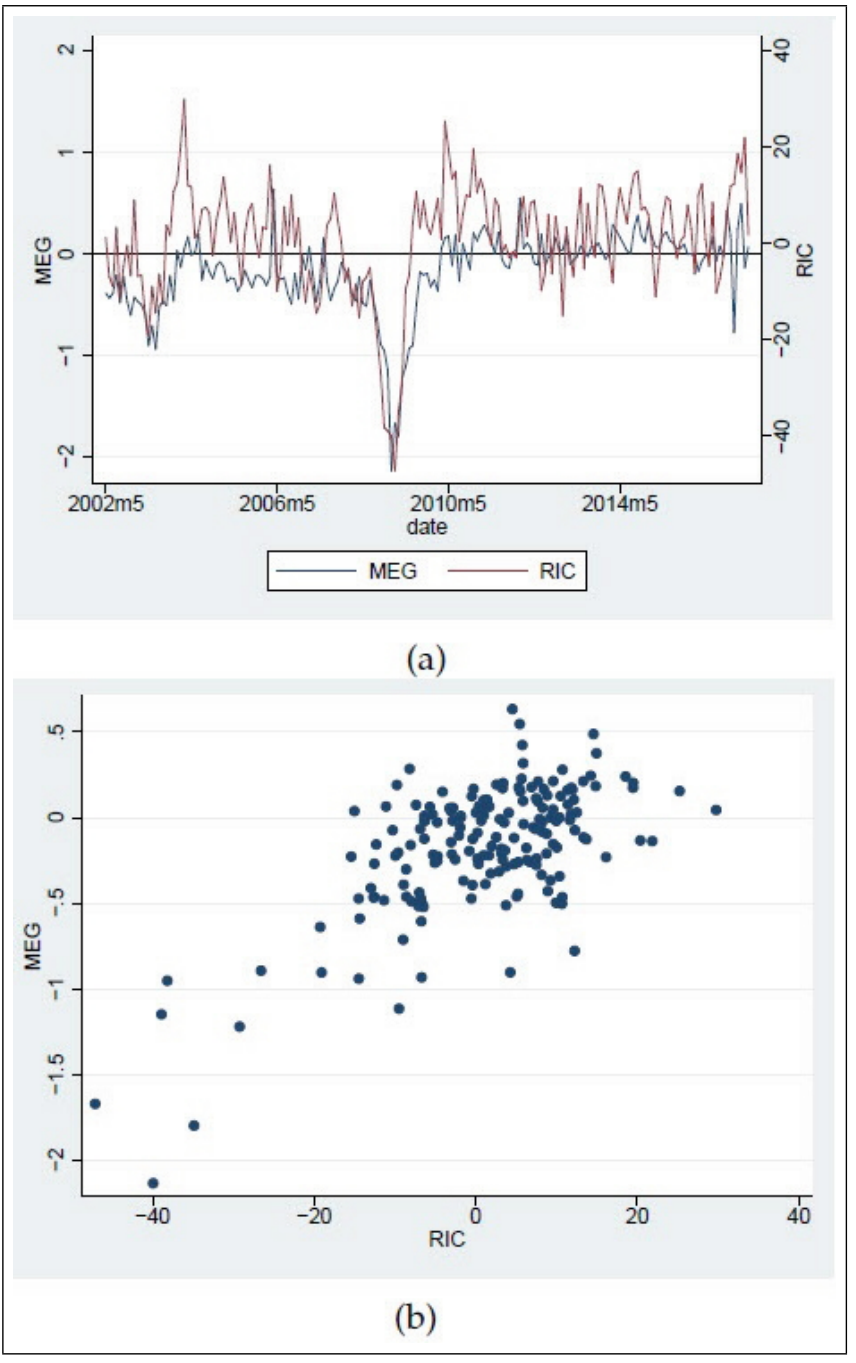

A key feature of the series considered in the analysis is that they exhibit high levels of persistence. ${ }^{11}$ This behavior is evident from the autocorrelation (ACF) and partial autocorrelation functions (PACF) of the series. Figure 4 shows the ACF and PACF of the $I S M_{t}, R I C_{t}$,

${ }^{11}$ We calculate and report in Table 11 in the Appendix the results of several unit root tests for all variables. In all cases, the tests reject the presence of a unit root. 
Figure 3 FRBR Diffusion Indices

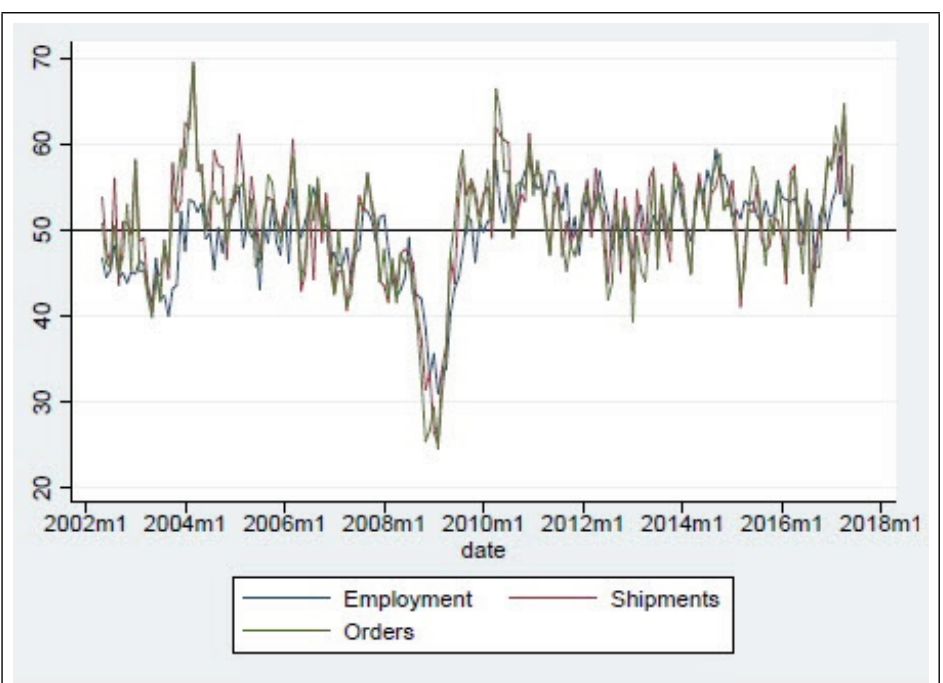

(a) Components of FRBR composite index
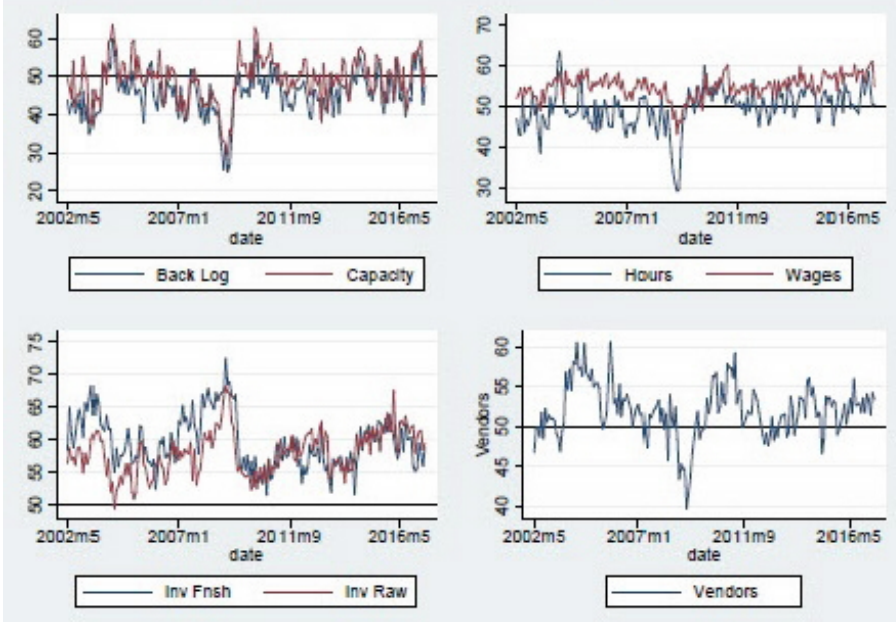

(b) Various FRBR individual diffusion indices

$M E G_{t}$, and the individual diffusion indices used to calculate $R I C_{t}$. In every case, the $\mathrm{ACF}$ and $\mathrm{PACF}$ indicate a strong autocorrelation at the first three of four lags. The latter is relevant because it suggests that when considering models that explain and predict the evolution of $I S M_{t}$ and $M E G_{t}$, it would become critical to incorporate their dy- 
Figure 4 ACF and PACF

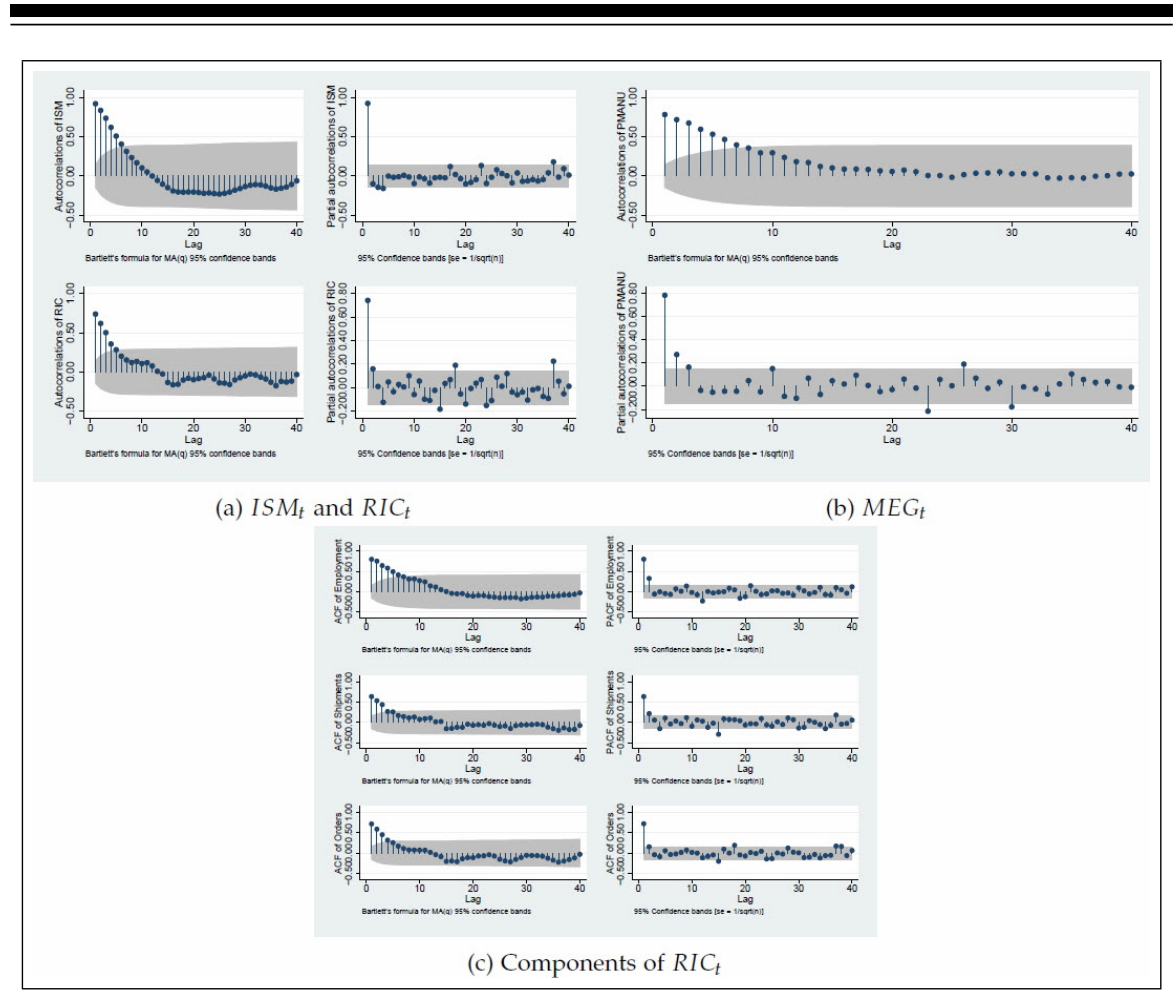

namic behavior in addition to the dynamic behavior of $R I C_{t}$ and $R I C_{t}^{i}$. We evaluate several univariate dynamic models that explain the behavior of the series in Sections 2.1 and 3.1.

\section{Cross-correlations}

As a first approximation to the analysis of the dynamic relationship between the FRBR diffusion indices and national and regional economic indicators, we examine the cross-correlations between the variables $X_{t}=I S M_{t}, M E G_{t}$, defined as $\operatorname{Corr}\left(X_{t}, R I C_{t+h}\right)$, for different values of $h=-20, \ldots,-1,0,1, \ldots 20$. The cross-correlograms between $I S M_{t}$ and $R I C_{t}$ and $M E G_{t}$ and $R I C_{t}$ are shown in Figure 5. Figure 5 a indicates a very strong contemporaneous correlation between $I S M_{t}$ and $R I C_{t}$, with a correlation equal to 0.80 (at $h=0$ ). The highest correlation between the $M E G_{t}$ and $R I C_{t}$ series, shown in Figure 5b, occurs at $h=-1$ and is equal to 0.68 . In other words, this last cross- 
Figure 5 Cross-correlograms

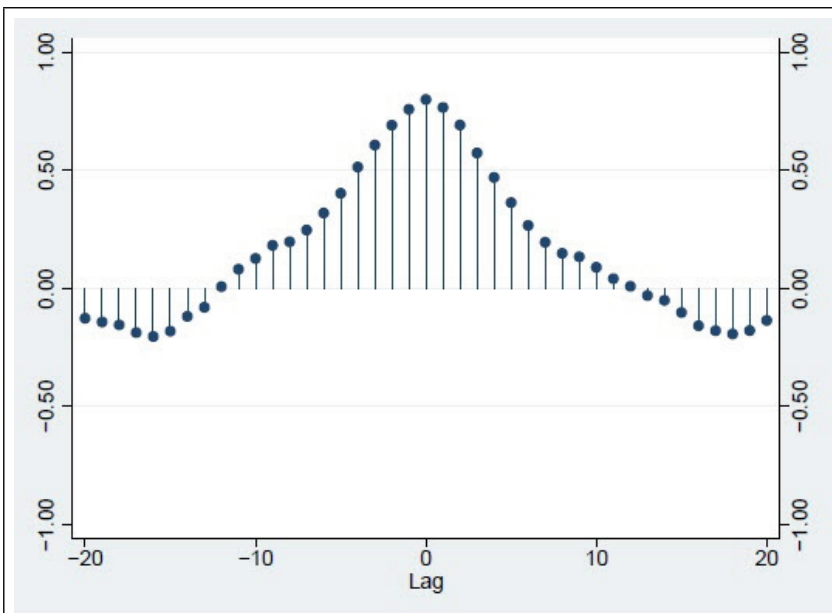

(a) $I S M_{t}$ and $R I C_{t}$

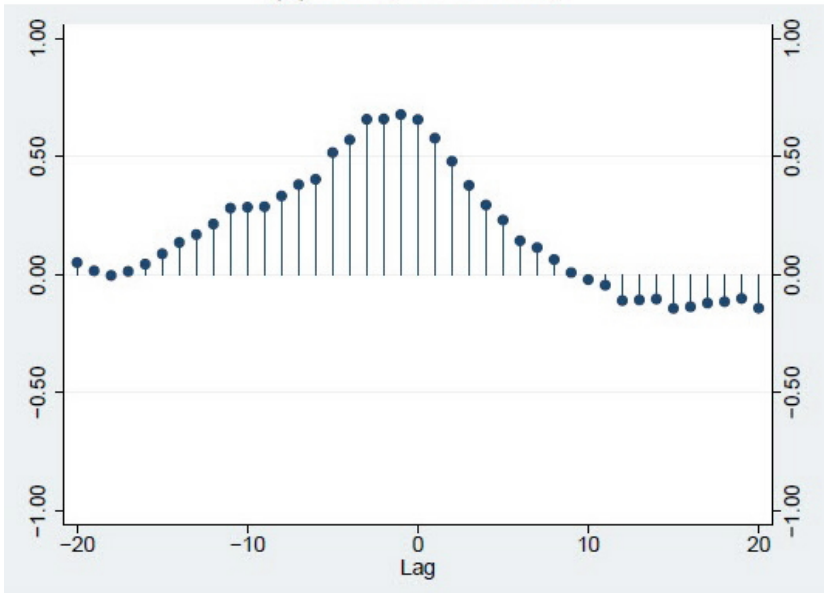

(b) $M E G_{t}$ and $R I C_{t}$

correlogram seems to indicate that $R I C_{t}$ tends to lead $M E G_{t}$, so $R I C_{t}$ may contain information about the future behavior of the $M E G_{t}$ series.

We also examine the cross-correlations between $I S M_{t}$ and $M E G_{t}$ and the individual FRBR diffusion indices, and among the individual FRBR diffusion indices themselves. The results, which are reported in Table 12 in the Appendix, can be summarized as follows. First, the cross-correlograms between $I S M_{t}$ and the FRBR individual diffusion indices $R I C_{t}^{i}$ generally reflect a strong contemporaneous relationship 
(at $h=0$ ), with the exception of $R I C_{t}^{E}$ and $R I C_{t}^{W}$ (in both cases, the highest correlation occurs at $h=1$; it is 0.71 for $R I C_{t}^{E}$ and 0.58 for $\left.R I C_{t}^{W}\right)$. Second, the highest correlation is between $I S M_{t}$ and $R I C_{t}^{V}$, with a value of 0.78 at $h=0$, followed by $R I C_{t}^{O}$, with a value of 0.77 also at $h=0$. Third, for some diffusion indices, specifically $R I C_{t}^{I F}$ and $R I C_{t}^{I R}$, the correlation is negative. Fourth, the results are somewhat different when comparing the cross-correlations between $M E G_{t}$ and $R I C_{t}^{i}$ in Table 13. For instance, the highest $\operatorname{Corr}\left(M E G_{t}, Y_{t+h}\right)$ is observed when $Y_{t+h}=R I C_{t+h}^{E}$ and $h=0$, with a value of 0.76 . Other diffusion indices, however, tend to lead $M E G_{t}$ series (when $Y_{t+h}=R I C_{t+h}^{O}$, the highest value is observed at $h=-3$, and when $Y_{t+h}=R I C_{t+h}^{S}$ the highest value is $\left.h=-1\right)$. Finally, the $R I C_{t}^{i}$ series also tend to move together. The cross-correlations between $R I C_{t}^{E}$, $R I C_{t}^{O}$, and $R I C_{t}^{S}$, and the other individual diffusion indices are reported in the Appendix in Tables 14, 15, and 16. Note that the correlations between $R I C_{t}^{E}$ and the other diffusion indices are relatively low. The correlations are higher for the series $R I C_{t}^{O}$ (for instance, the contemporaneous correlations between $R I C_{t}^{O}$ and $R I C_{t}^{S}$ and between $R I C_{t}^{O}$ and $R I C_{t}^{C}$ are, respectively, 0.92 and 0.90 ). The main takeaway from this preliminary analysis is that the information contained in the FRBR survey seems to be highly correlated with changes in both national and regional economic conditions. However, based on the correlations observed between the series, various composite indices based on different series and weights may be constructed to more accurately explain national and regional economic changes.

\section{PREDICTING THE NATIONAL ECONOMY}

We now proceed to a formal analysis of how well the FRBR composite index $R I C_{t}$ tracks the national economy. As mentioned earlier, we use the $I S M_{t}$ series as a gauge of national economic activity. We begin by first evaluating the predictive ability of a number of univariate dynamic models of $I S M_{t}$ for benchmarking purposes. We next estimate several linear and vector autoregressive models (VARs) that incorporate the diffusion indices obtained from the FRBR manufacturing survey, and we examine how this additional information improves the models' predictive ability. We specifically compare the predictive power of models that use the composite diffusion index $R I C_{t}$ to other less-constrained models in which the components of $R I C_{t}$ are considered individually, as well as models that incorporate other diffusion indices, not part of $R I C_{t}$, calculated from currently available FRBR survey data. 


\section{Univariate models of ISM}

The descriptive analysis of Section 1.1 suggests that the $I S M_{t}$ series is highly persistent. In order to formally examine its behavior, we first estimate several univariate dynamic models of $I S M_{t}$ and determine how well these models predict $I S M_{t}{ }^{12}$ These univariate dynamic models are used as the benchmark against which we evaluate the performance of models that incorporate the information from the FRBR survey. We consider models that assume a general $\operatorname{ARMA}(p, q)$ representation of the form

$$
I S M_{t}=a+\sum_{j=1}^{p} \phi_{j} I S M_{t-j}+\varepsilon_{t}+\sum_{j=1}^{q} \theta_{j} \varepsilon_{t-j},
$$

where $\varepsilon_{t}$ is assumed to be an i.i.d. white noise process, and $\phi=$ $\left[\phi_{1}, \ldots, \phi_{p}\right]$ and $\theta=\left[\theta_{1}, \ldots, \theta_{q}\right]$ are the autoregressive and moving average coefficients, respectively. Table 17 in the Appendix presents estimates of several univariate models fitted to the $I S M_{t}$ series, together with the goodness-of-fit statistics AIC and BIC. Based on the estimation results, $\mathrm{AR}(4)$ has the best AIC statistics, whereas $\mathrm{AR}(1)$ produces the best BIC statistics. ${ }^{13}$ These models produce residuals with RMSE of 1.841 for $\mathrm{AR}(1)$ and 1.789 for $\mathrm{AR}(4)$. Figure 6 shows one-step-ahead predictions of $I S M_{t}$ for the AR(1) and AR(4) models. Overall, the results suggest that past values of $I S M_{t}$ explain fairly well the behavior of the series. The contribution of the information contained in the FRBR survey should, therefore, be assessed by comparing different models that include the FRBR diffusion indices to the performance of these very simple univariate dynamic models.

\section{Linear models}

We now present estimates of several linear models that explain the behavior of $I S M_{t}$ using the information collected from the FRBR survey series. First, we consider very simple models that assume a contemporaneous relationship between the variables of the form

$$
I S M_{t}=\alpha+X_{t} \beta+\varepsilon_{t},
$$

where $X_{t}$ is a vector of regional predictors and $\varepsilon_{t}$ is an error term assumed to be an i.i.d. white noise process. Table 2 presents the estimates of four alternative model specifications depending on the variables

\footnotetext{
12 Throughout the paper, we compare models based on their predictive accuracy measured by the root mean squared error (RMSE).

${ }^{13}$ Several alternative $\operatorname{ARMA}(p, q)$ were estimated; those reported in Table 17 have the smallest $\mathrm{AIC}$ and BIC statistics.
} 
Figure 6 One-Step-Ahead Predictions of $I S M_{t}$

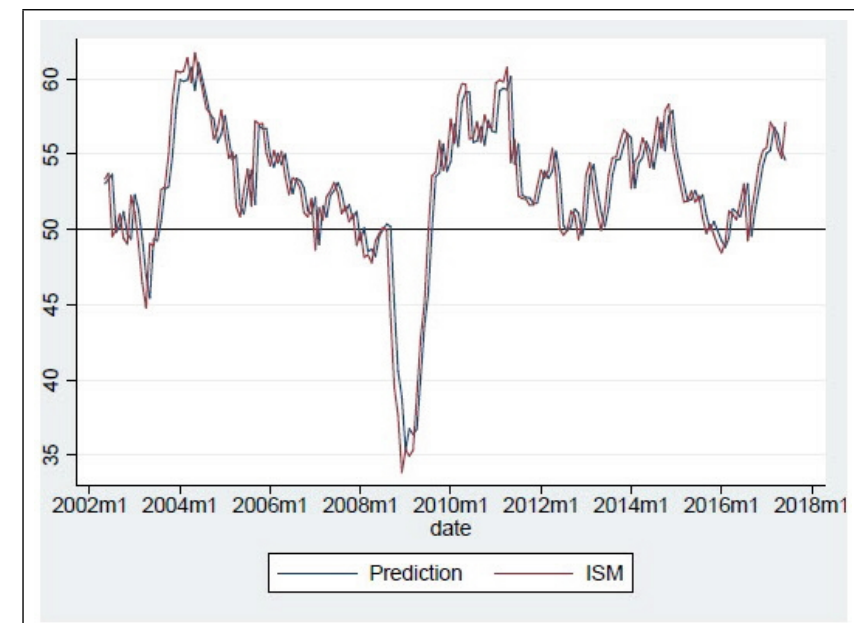

(a) $\operatorname{AR}(1)$ model

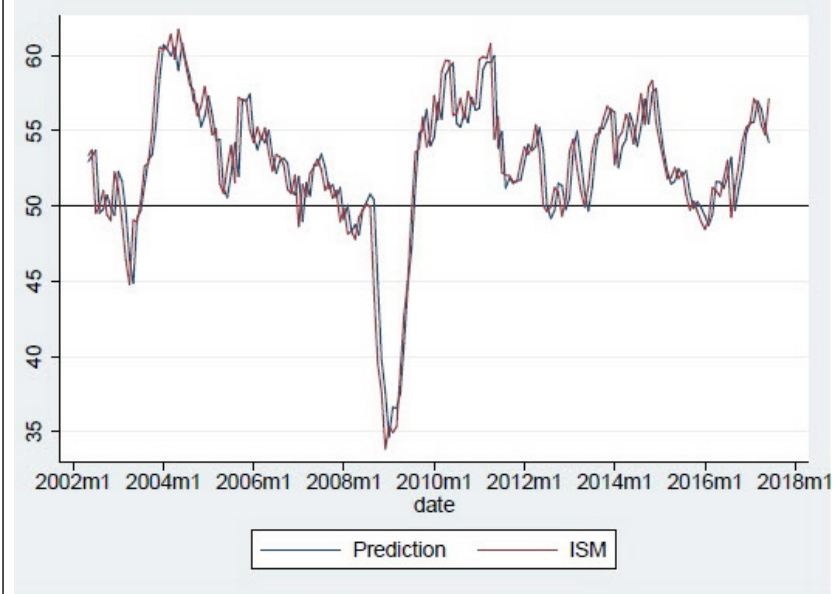

(b) AR(4) model

included in $X_{t}$. Model (1) includes only the composite index of the FRBR series constructed as a weighted average of $R I C_{t}^{E}, R I C_{t}^{S}$, and $R I C_{t}^{O}$, given by (2); in other words, $X_{t}=R I C_{t}$ in this case. In model (2), each one of the components of the composite index are included in an unconstrained form, meaning that $X_{t}=\left[R I C_{t}^{E}, R I C_{t}^{S}, R I C_{t}^{O}\right]$. Model (3) adds additional components not used in the composite index but available in the FRBR survey, and model (4) is basically a 
Table 2 Linear Models of ISM: Contemporaneous Regressors

\begin{tabular}{|c|c|c|c|c|}
\hline$R I C_{t}$ & $\begin{array}{l}(1) \\
0.665^{* * *} \\
(0.054)\end{array}$ & (2) & (3) & (4) \\
\hline$R I C_{t}^{E}$ & & $\begin{array}{l}0.269^{* *} \\
(0.089)\end{array}$ & $\begin{array}{l}0.143^{*} \\
(0.068)\end{array}$ & $\begin{array}{l}0.149^{*} \\
(0.064)\end{array}$ \\
\hline$R I C_{t}^{S}$ & & $\begin{array}{l}0.184^{*} \\
(0.089)\end{array}$ & $\begin{array}{l}0.106 \\
(0.078)\end{array}$ & \\
\hline$R I C_{t}^{O}$ & & $\begin{array}{l}0.246^{*} \\
(0.101)\end{array}$ & $\begin{array}{l}0.096 \\
(0.096)\end{array}$ & $\begin{array}{l}0.168^{* * *} \\
(0.048)\end{array}$ \\
\hline$R I C_{t}^{B}$ & & & $\begin{array}{l}0.037 \\
(0.070)\end{array}$ & \\
\hline$R I C_{t}^{C}$ & & & $\begin{array}{l}-0.061 \\
(0.089)\end{array}$ & \\
\hline$R I C_{t}^{V}$ & & & $\begin{array}{l}0.479^{* * *} \\
(0.087)\end{array}$ & $\begin{array}{l}0.521^{* * *} \\
(0.082)\end{array}$ \\
\hline$R I C_{t}^{H}$ & & & $\begin{array}{l}-0.007 \\
(0.071)\end{array}$ & \\
\hline$R I C_{t}^{W}$ & & & $\begin{array}{l}0.083 \\
(0.095)\end{array}$ & \\
\hline$R I C_{t}^{I V}$ & & & $\begin{array}{l}-0.184^{*} \\
(0.072)\end{array}$ & $\begin{array}{l}-0.193^{* *} \\
(0.067)\end{array}$ \\
\hline$R I C_{t}^{I R}$ & & & $\begin{array}{l}-0.239^{* *} \\
(0.078)\end{array}$ & $\begin{array}{l}-0.220^{* *} \\
(0.074)\end{array}$ \\
\hline Constant & $\begin{array}{l}19.282^{* * *} \\
(2.762)\end{array}$ & $\begin{array}{l}17.618^{* * *} \\
(3.898)\end{array}$ & $\begin{array}{l}32.422^{* * *} \\
(8.178)\end{array}$ & $\begin{array}{l}34.024^{* * *} \\
(7.659)\end{array}$ \\
\hline$N$ & 182 & 182 & 182 & 182 \\
\hline $\operatorname{Adj}-R^{2}$ & 0.638 & 0.639 & 0.768 & 0.770 \\
\hline RMSE & 2.893 & 2.889 & 2.318 & 2.307 \\
\hline
\end{tabular}

Note: Newey-West standard errors in parentheses; ${ }^{*} p<0.05,{ }^{* *} p<0.01,{ }^{* * *} p<$ 0.001 .

refinement of model (3) obtained through a stepwise procedure of regressor selection. ${ }^{14}$

${ }^{14}$ Throughout the paper, we follow a standard stepwise procedure to select the variables of the model. We typically proceed from general to particular: we start with a general model that includes the largest possible set of predictors (in the dynamic versions of the models, we also include lagged values of the variables), then we remove predictors with the highest p-values and refit the model. The procedure also takes into account, when comparing models, their respective AIC and BIC values. Standard errors are produced by a Newey-West regression procedure that corrects potential serial correlation in the error terms. While under serial correlation, OLS still produces unbiased parameter estimates, the standard errors in this case are not efficient. We reestimate the model in (5) using the Newey-West regression procedure that produces serial correlation robust standard errors. The adjusted- $R^{2}$ measure is from the OLS regression. 
The results show that the behavior of the FRBR series explains considerable variation of $I S M_{t}$. Specifically, model (1) shows that the FRBR composite index $R I C_{t}$ explains, by itself, about 64 percent of variation of $I S M_{t}$. Model (1) also tells us that when $R I C_{t}=50$, which represents the point at which the percentage of respondents reporting an increase is the same as the percentage reporting a decrease in the FRBR survey, the ISM composite index is 52.53. According to the linear estimates, $R I C_{t}$ and $I S M_{t}$ are equal when $R I C_{t}$ is approximately 57. Moreover, when $R I C_{t}$ is higher (lower) than 57 , then $R I C_{t}>(<$ )ISM .

We additionally perform the following exercise. Suppose the goal is to construct a composite index $\overline{R I C}_{t}$ that includes $\left\{R I C_{t}^{E}, R I C_{t}^{S}\right.$, $\left.R I C_{t}^{O}\right\}$ and tracks as closely as possible the $I S M_{t}$ series. Specifically, suppose that $\overline{R I C}_{t}$ takes the functional form $\overline{R I C}_{t}=\alpha+\beta^{E} R I C_{t}^{E}+$ $\beta^{E} R I C_{t}^{S}+\beta^{E} R I C_{t}^{O}$, and $\left\{\alpha, \beta^{E}, \beta^{S}, \beta^{O}\right\}$ are chosen so as to minimize $\sum_{t=1}^{T}\left(I S M_{t}-\overline{R I C}_{t}\right)^{2}$, subject to the constraints $\beta^{E}+\beta^{S}+\beta^{O}=1$, $\beta_{i} \geq 0$. The values obtained in this case are: $\alpha=2.545, \beta^{O}=0.53$, $\beta^{S}=0.33, \beta^{E}=0.14$. Two remarks are worth making. First, since the $I S M_{t}$ series seems to be displaced upward, as explained before, $\overline{R I C}_{t}$ includes a positive constant term (note that the current composite FRBR diffusion index $R I C_{t}$ does not have a constant term). Second, $R I C_{t}^{O}$ should receive the highest weight and $R I C_{t}^{E}$ the lowest weight in the composite index, if the objective is to construct a composite index that tracks as closely as possible the $I S M_{t}$ series.

When each individual component is included as separate regressors in an unconstrained way (model [2]), the fit and predictive power of the model improve, but note that such improvement is relatively small. Also, by comparing the estimates of model (2), $R I C_{t}^{E}$ seems to be the most important variable at explaining the behavior of $I S M_{t}$, but in the construction of the composite index $R I C_{t}$, this variable receives the lowest weight of the three individual diffusion indices. Sizable improvements in fit and predictive ability are observed, however, when we incorporate additional survey information, as evidenced by models (3) and (4). In general, the results of Table 2 confirm that when the objective is to describe or predict the evolution of the national economy, including other information readily available through the FRBR survey would tend to improve the outcome.

Including only contemporaneous values of the FRBR diffusion indices is somewhat restrictive. It is clear from Section 1.1 that the series show high levels of persistence, which suggests that further improvements could be obtained by using models that include a dynamic structure. Thus, we estimate next a set of models that account for this 
more general dynamic behavior of the form

$$
I S M_{t}=\alpha+\sum_{j=0}^{3} \beta_{j} X_{t-j}+\sum_{j=1}^{3} \gamma_{j} I S M_{t-j}+\varepsilon_{t},
$$

where $\varepsilon_{t}$ is again assumed to be an i.i.d. distributed white noise process. Table 3 presents the estimates of four models of the type represented by (6): model (1) includes contemporaneous and lagged value of the FRBR composite index $R I C_{t}$; model (2) adds lagged values of $I S M_{t}$; model (3) includes contemporaneous and lagged values of the components of the $R I C_{t}$; and model (4) includes lagged values of $I S M_{t} .{ }^{15}$

By simply considering lagged values of $R I C_{t}$, such as in model (1), the RMSE decreases substantially (the static model [1] of Table 2 has a RMSE equal to 2.893, and this one has a RMSE of 2.539). However, once the model incorporates lagged values of $I S M_{t}$, such as in model (2), the explanatory power of $R I C_{t}$ declines. Also, model (2) has a much better predictive accuracy. Model (3) is an improvement relative to model (1) but not relative to model (2). Considering both a less constrained and richer dynamic behavior undoubtedly increases the fit of the model and improves its predictive power. Model (4), which includes lagged values of the individual diffusion indices $R I C_{t}^{i}$, $i=E, O, S$, and lagged values of $I S M_{t}$, has the lowest RMSE among all models, with a value of 1.676. Notice that the FRBR diffusion index that captures changes in orders, $R I C_{t}^{O}$, is always relevant at explaining the behavior of $I S M_{t}$, even after accounting for past values of $I S M_{t}$.

Finally, Table 4 shows the estimates of a model obtained by a stepwise procedure of regressor selection among the FRBR survey series $R I C_{t}^{i}$, and their respective lags, and lagged values of $I S M_{t}$. In this case, the model explains almost 90 percent of the variation in $I S M_{t}$. The variables that seem to be most relevant at explaining changes in $I S M_{t}$ include, in addition to $I S M_{t-1}$, the regional indicators $R I C_{t-2}^{E}$, $R I C_{t-2}^{O}$, and $R I C_{t}^{V}$. The linear predictions of this model are shown in Figure 7. The RMSE is 1.554, and this value is the lowest among all the models considered up to this point.

\section{Vector autoregressive models}

In this section, we use VAR models to further explore and understand the relationship between the $I S M_{t}$ series and the indices elaborated by the FRBR. Let $Z_{t}=\left[R I C_{t}^{1}, R I C_{t}^{2}, \cdots, R I C_{t}^{m}, I S M_{t}\right]$ be a multivariate

\footnotetext{
${ }^{15}$ Linear predictions of models (1) through (4) are shown in Figure 16 in Appendix
} A.3.2. 
Table 3 Linear Models of ISM: Contemporaneous Lagged Regressors

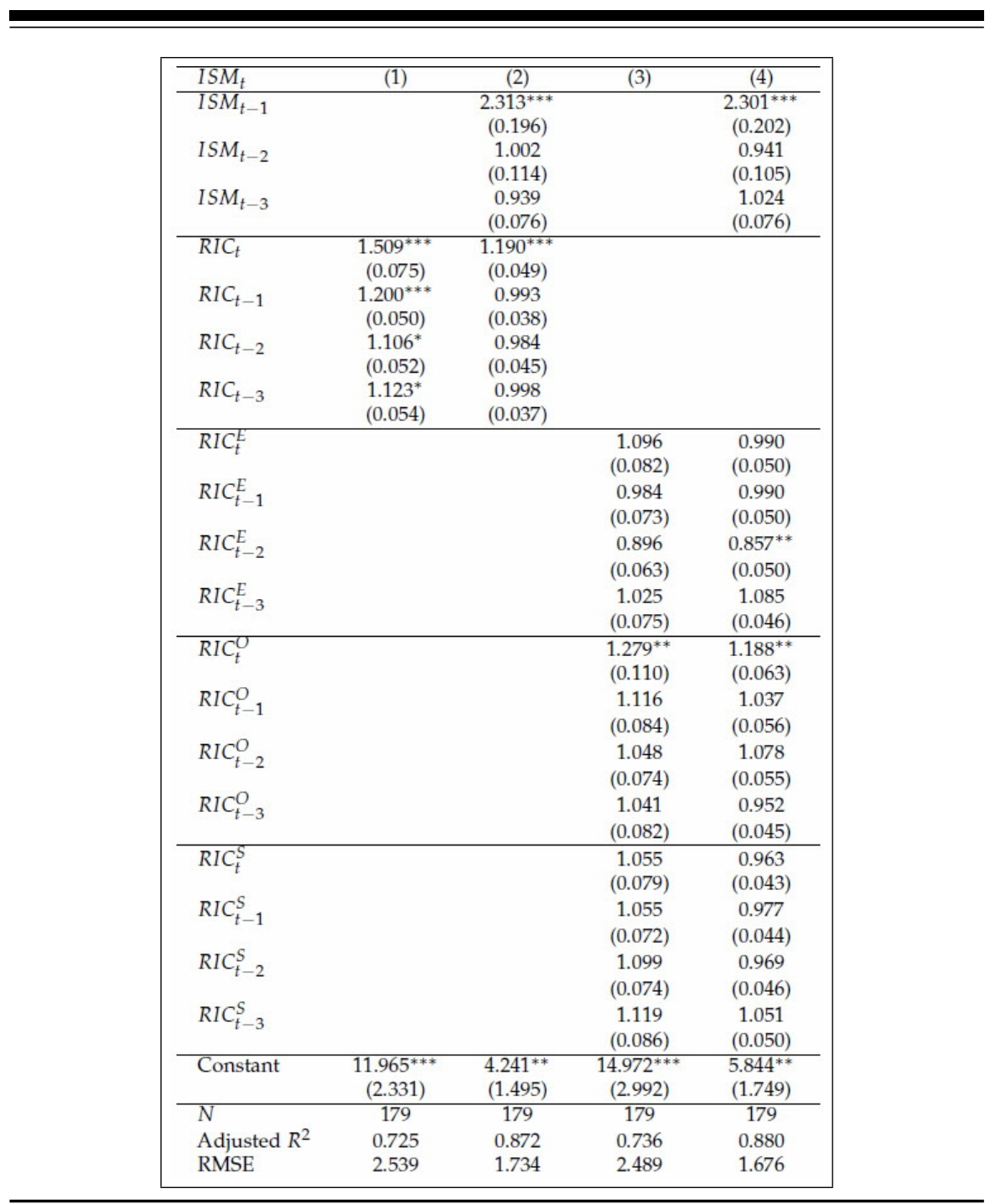

Note: Newey-West standard errors in parentheses; ${ }^{*} p<0.05,{ }^{* *} p<0.01,{ }^{* * *} p<$ 0.001 .

time series, where $R I C_{t}^{i}$ represents each one of the diffusion indices at time $t$. The long-run structural relationship between the FRBR series 
Table 4 Linear Model of ISM: Stepwise Selection

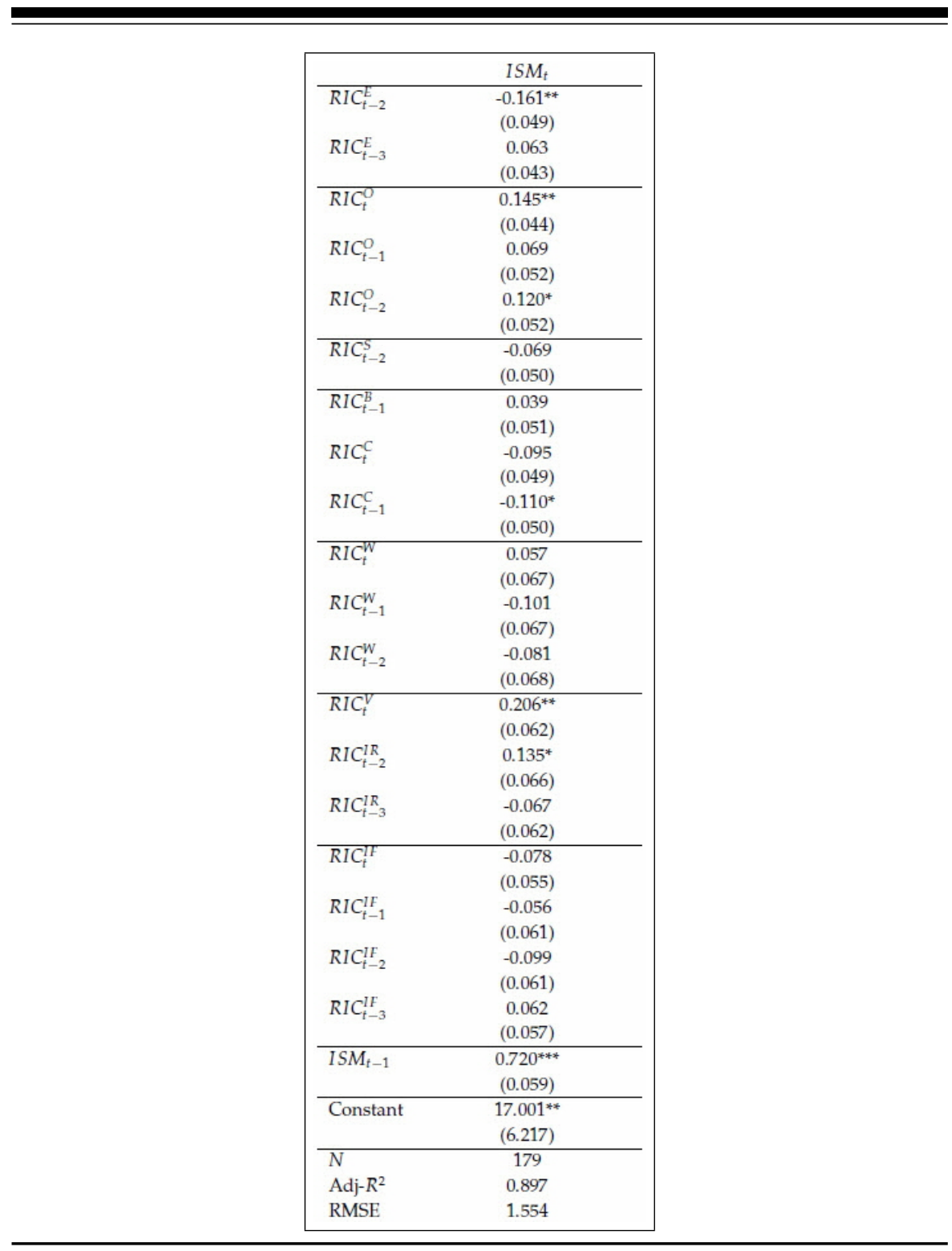

Note: Newey-West standard errors in parentheses; ${ }^{*} p<0.05,{ }^{* *} p<0.01,{ }^{* * *} p<$ 0.001 .

and $I S M_{t}$ is modeled by the $p^{t h}$-order VAR process

$$
B Z_{t}=a+\sum_{j=1}^{p} A_{j} Z_{t-j}+\varepsilon_{t},
$$


Figure 7 Stepwise Selection Model of ISM: Observed Values and Predictions

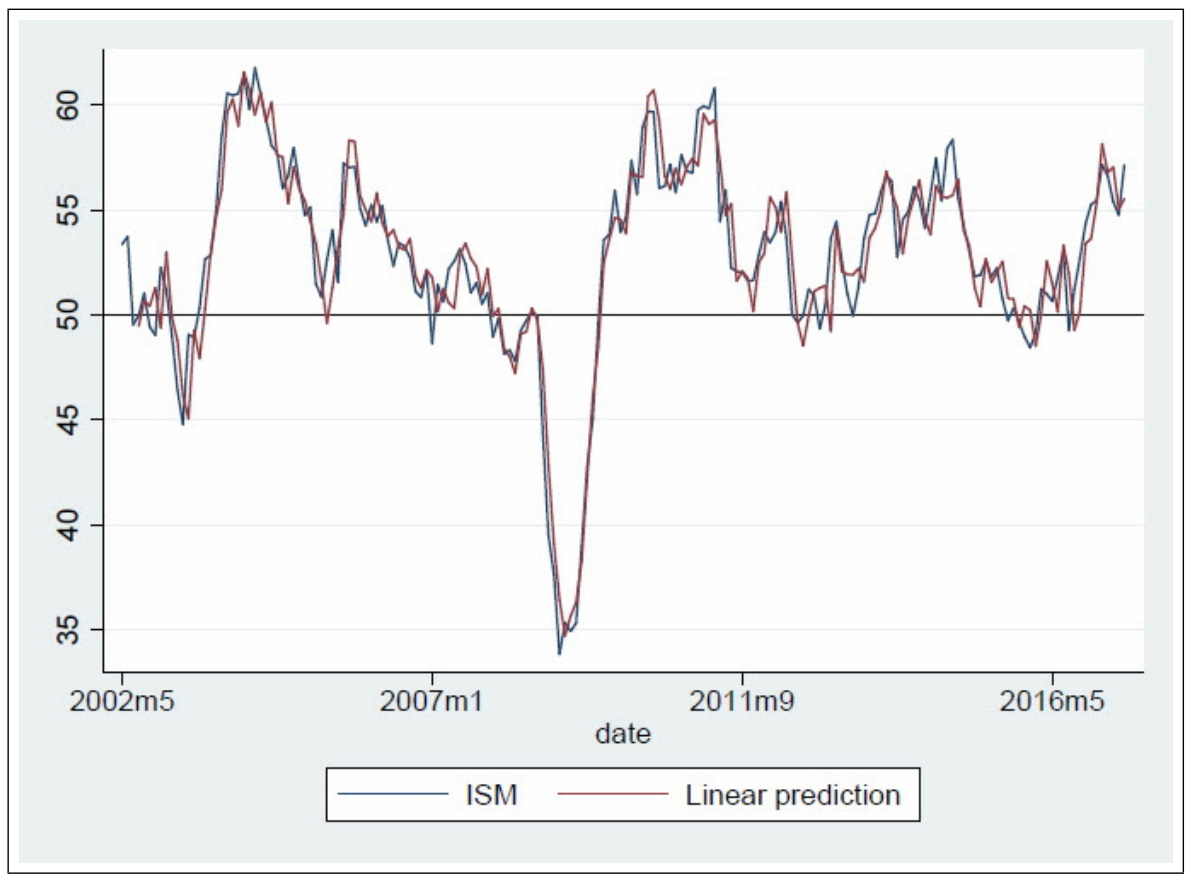

where $B$ and $A_{j}$ are $(m+1) \times(m+1)$ matrices, and $\varepsilon_{t}=\left[\varepsilon_{t}^{1}, \varepsilon_{t}^{2}, \ldots, \varepsilon_{t}^{m+1}\right]^{\prime}$ is a multivariate white noise process with mean zero and variance $I_{(m+1) \times(m+1)}$. Multiplying both sides of $(7)$ by the inverse of $B$, we obtain

$$
Z_{t}=\alpha+\sum_{j=1}^{p} \Phi_{j} Z_{t-j}+e_{t}
$$

where $\alpha=B^{-1} a, \Phi_{j}=B^{-1} A_{j}$ and $e_{t}=\left[e_{t}^{1}, e_{t}^{2}, \ldots, e_{t}^{m+1}\right]^{\prime}$ is a multivariate mean zero white noise process with variance-covariance matrix $\Sigma_{e}=B^{-1}\left(B^{-1}\right)^{\prime}$. The equation in (8) represents a VAR model of order $p$, which can be estimated by maximum likelihood. With the estimates of the $\Sigma_{e}$ matrix, we perform a Cholesky decomposition and obtain a lower triangular matrix $P$ such that $\Sigma_{e}=P P^{\prime}$. Premultiplying (8) by $P^{-1}$ yields

$$
P^{-1} Z_{t}=P^{-1} \alpha+P^{-1} \sum_{j=1}^{p} \Phi_{j} Z_{t-j}+u_{t}
$$


where $u_{t}=P^{-1} e_{t}$ is a multivariate white noise process with variancecovariance matrix $I_{(m+1) \times(m+1)}$. The expression in (9) gives us $(m+1)$ equations in the FRBR series and $I S M_{t}$, in addition to their past values. Because $P$ is lower triangular, so is $P^{-1}$, thus the $(m+1)$-st equation of (9) contains all current and past values of the multivariate time series. Also note that the error term $u_{t}^{m+1}$ is the linear combination of error terms $\left(e_{t}^{1}, e_{t}^{2}, \ldots, e_{t}^{m+1}\right)$ weighted by the coefficients of the matrix $P^{-1}$. The expression obtained in the $(m+1)$-st equation is what is known as the structural equation of $I S M_{t}$. This equation represents $I S M_{t}$ as a linear function of its past values (up to $p$-th lag), as well as contemporaneous and lagged values of the FRBR series. Using this equation, we then construct "predictions" of the value of the ISM diffusion index under the following premise: at time $t$, when regional survey results have become known, we can use this information to obtain a reasonable prediction of the value of the ISM diffusion index for the current time period.

\section{Bivariate VAR model: ISM and FRBR composite diffusion indices}

We first estimate a $\operatorname{VAR}(1)$ model for bivariate series consisting of the composite diffusion indices $I S M_{t}$ and $R I C_{t}$. The selection of lags is based on AIC and BIC statistics. The parameter estimates of the VAR(1) model are shown in Table 18 in the Appendix, together with the variance-covariance matrix of the error terms and its Cholesky decomposition. ${ }^{16}$ Using the inverse of the lower triangular matrix obtained from the Cholesky decomposition, we construct the structural form for $I S M_{t}$, which is plotted in Figure 17 in the Appendix. The RMSE of this specification is 1.72. We additionally perform a forecast error variance decomposition (FEVD) to interpret the results of the VAR model. The FEVD quantifies the relative contribution of the variables in the system, in this case ISM and RIC, to the variance of the forecast error of each variable. We focus here on the forecast error variance of $I S M_{t}$. The top panel in Figure 8 shows the percentage of the forecast error variance of ISM explained by RIC, and the bottom panel shows the percentage explained by itself. The figure indicates that variations in ISM are mostly explained by shocks to the series itself; the variation explained by RIC is virtually zero.

\footnotetext{
${ }^{16}$ The table and the figure showing the observed and predicted values are shown in Appendix A.3.3.
} 
Figure 8 VAR Model: $I S M_{t}$ and $R I C_{t}$. Forecast Error Variance Decomposition

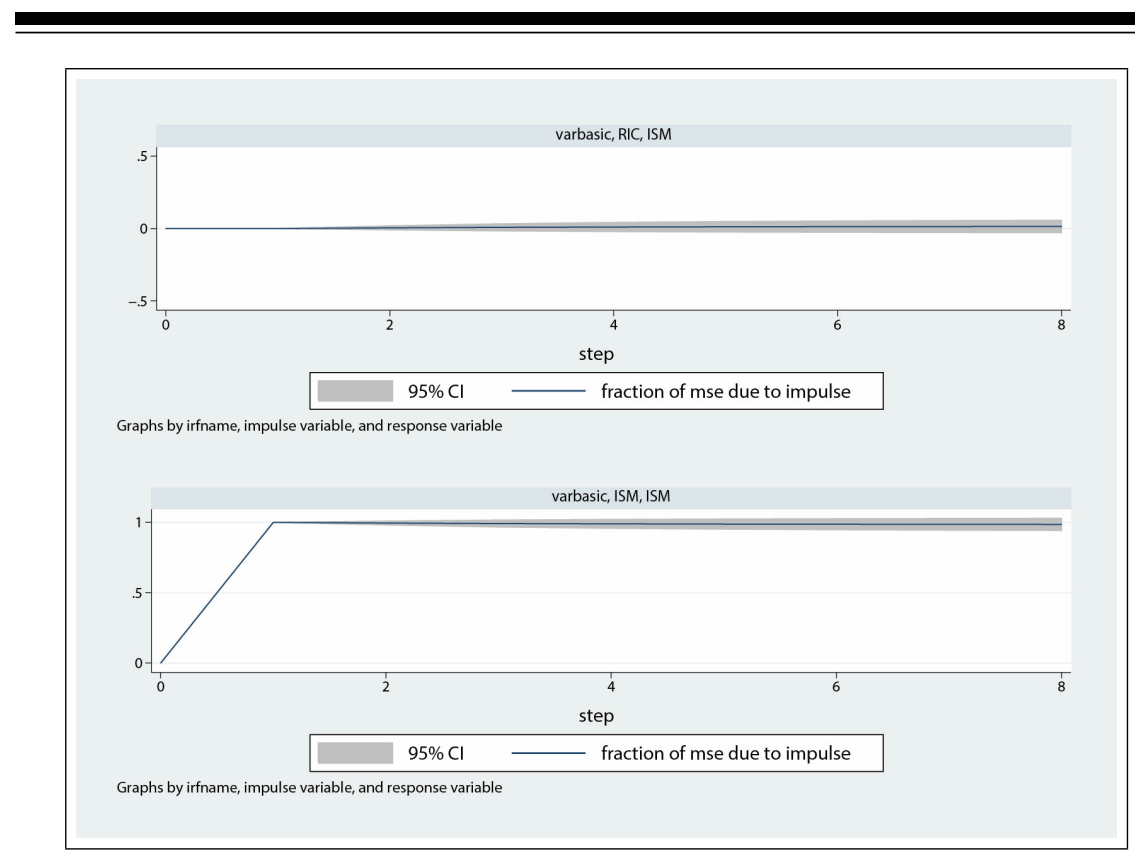

\section{Multivariate VAR: ISM and FRBR individual diffusion indices}

We now estimate a VAR model that includes $I S M_{t}$ and the individual diffusion indices used in the FRBR composite index, $R I C_{t}$. The AIC and BIC statistics suggest that a $\operatorname{VAR}(2)$ model fits the data best. The parameter estimates of the $\operatorname{VAR}(2)$ model are shown in Table 19 in Appendix A.3.4, along with the variance-covariance matrix and its Cholesky decomposition. Using the inverse of the lower triangular matrix obtained from the Cholesky decomposition, we construct the structural form of $I S M_{t} .{ }^{17}$ The prediction errors have a standard deviation of 1.64 , which is slightly smaller than the RMSE of the bivariate VAR model considered in the previous section. The FEVD in Figure 9 describes the effect of a shock on the variables $R I C^{E}, R I C^{S}, R I C^{O}$, and ISM on the forecast error variance of ISM. Once again, the figure indicates that shocks on the variable ISM essentially explain most of

\footnotetext{
${ }^{17}$ Figure 18 in Appendix A.3.4 compares the predicted values of the model to the observed values.
} 


\section{Figure 9 ISM and FRBR Individual Diffusion Indices. Forecast Error Variance Decomposition}

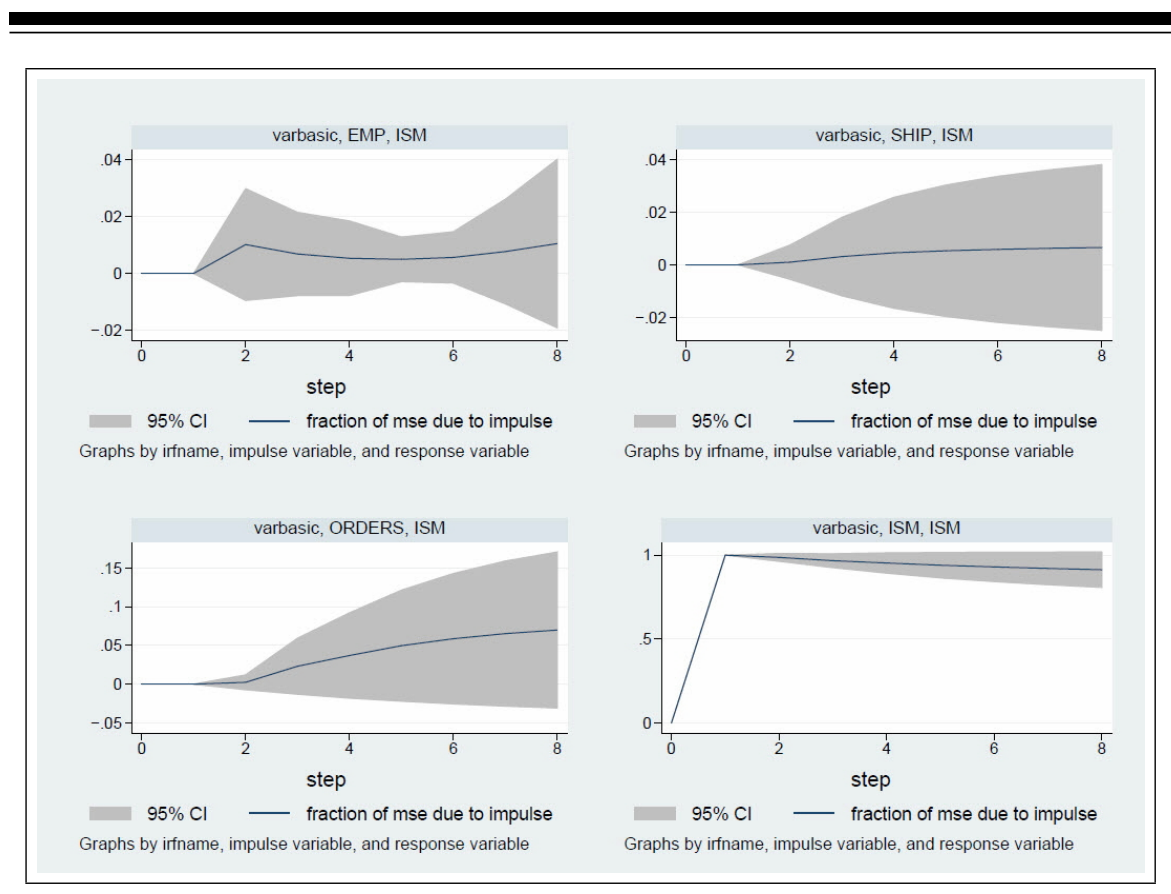

the variation of ISM. However, the variable $R I C^{O}$ now becomes relevant, explaining approximately 8 percent of the variation in ISM after eight periods.

We finally estimate a VAR model that incorporates other diffusion indices available from the FRBR survey. The variables included in the analysis were selected through a stepwise regression procedure similar to the one followed in Section 2.2. Based on AIC and BIC statistics, the VAR(1) model fits the data best. The results are reported in Table 20 in Appendix A.3.4. This specification offers a high level of predictive accuracy with the lowest RMSE, which is equal to 0.85. The FEVD in Figure 10 confirms the importance of the ISM series in explaining its own variation. The FEVD also shows that, among all FRBR diffusion indices, $R I C^{O}$ is the most important one for explaining variations in ISM. 


\section{Figure 10 ISM and FRBR Individual Diffusion Indices. Forecast Error Variance Decomposition}

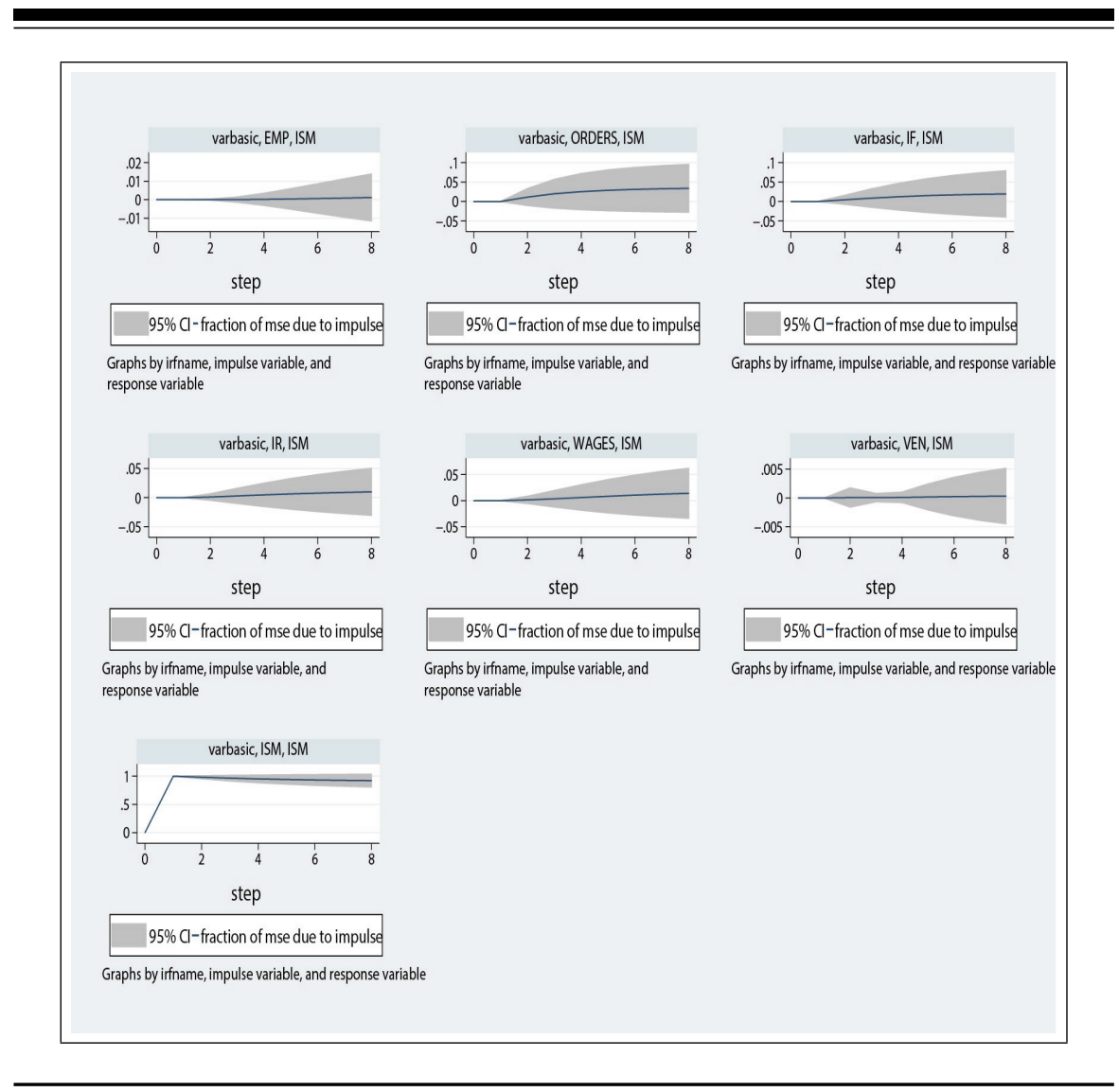

\section{Summary of findings}

To summarize our findings, in Table 5 we compare the RMSE of the models discussed thus far. We include, for comparison, the RMSE of the model that includes only the composite index $R I C_{t}$ currently reported by the FRBR. In light of the predictive accuracy of the models, it is clear that a multivariate VAR model dominates all other alternatives, producing a RMSE equal to 0.85 . However, a linear dynamic model that considers information readily available through the FRBR survey but not currently included in the calculation of the composite index $R I C_{t}$, offers more accurate predictions, with a RMSE of 1.55. So even the consideration of this last relatively simpler model would entail an important increase in predictive accuracy compared with a 
Table 5 RMSE for Selected Models of ISM

\begin{tabular}{|c|c|c|}
\hline & & RMSE \\
\hline Composite index & $R I C_{t}$ & 2.89 \\
\hline Univariate & $\begin{array}{l}A R(1) \\
A R(4)\end{array}$ & $\begin{array}{l}1.84 \\
1.79\end{array}$ \\
\hline Linear & $\begin{array}{l}\text { Contemporaneous } \\
\text { Dynamic }\end{array}$ & $\begin{array}{l}2.31 \\
1.55\end{array}$ \\
\hline VAR & $\begin{array}{l}\text { Bivariate } \\
\text { Multivariate }\end{array}$ & $\begin{array}{l}1.72 \\
0.85\end{array}$ \\
\hline
\end{tabular}

model that relies exclusively on the composite index $R I C_{t}$, which has the highest RMSE, equal to 2.89 .

\section{PREDICTING THE REGIONAL ECONOMY}

While in Section 2 we examined the extent to which the information collected by the FRBR manufacturing survey helps explain changes in the national economy, we now focus on how well the survey tracks the regional economy. As explained earlier, we use payroll MEG as a measure of regional manufacturing activity for two reasons. First, MEG data are available monthly and for all states. Second, MEG is a good indicator of the economic performance of the manufacturing sector, which is the focus of the present analysis, so MEG serves as a reasonable benchmark against which to assess the predictive ability of the information contained in the FRBR manufacturing survey. Our approach is similar to that in the previous section: we begin by estimating several univariate dynamic models of MEG; next, we compare the performance of these models to the performance of linear and VAR models of MEG that incorporate the diffusion index series from the FRBR manufacturing survey.

\section{Univariate models of MEG}

We first examine the predictive power of simple univariate dynamic models that only include the MEG series. The inspection of the MEG autocorrelation and partial autocorrelation functions in Figure 4 reveal that the series show high levels of persistence. To capture such dynamic behavior more formally, we estimate, as we did earlier with the ISM 
Table 6 Univariate Models of MEG

\begin{tabular}{ccccccc}
\hline MEG & $(1)$ & $(2)$ & $(3)$ & $(4)$ & $(5)$ & $(6)$ \\
& AR $(2)$ & AR $(3)$ & $\begin{array}{c}\text { ARMA } \\
(1,1)\end{array}$ & $\begin{array}{c}\text { ARMA } \\
(1,2)\end{array}$ & $\begin{array}{c}\text { ARMA } \\
(2,1)\end{array}$ & $\begin{array}{c}\text { ARMA } \\
(2,2)\end{array}$ \\
\hline$\phi_{1}$ & $0.569^{* * *}$ & $0.526^{* * *}$ & $0.915^{* * *}$ & $0.910^{* * *}$ & $0.862^{* * *}$ & 0.300 \\
& $(0.049)$ & $(0.054)$ & $(0.033)$ & $(0.042)$ & $(0.200)$ & $(1.522)$ \\
$\phi_{2}$ & $0.271^{* * *}$ & $0.177^{* *}$ & & & 0.0459 & 0.565 \\
& $(0.051)$ & $(0.065)$ & & & $(0.157)$ & $(1.381)$ \\
$\phi_{3}$ & & $0.162^{*}$ & & & & \\
& & $(0.066)$ & & & & \\
$\theta_{1}$ & & & $-0.374^{* * *}$ & $-0.386^{* * *}$ & -0.331 & 0.251 \\
& & & $(0.069)$ & $(0.071)$ & $(0.198)$ & $(1.525)$ \\
$\theta_{2}$ & & & & 0.0328 & & -0.253 \\
& & & & $(0.065)$ & & $0.534)$ \\
Constant & -0.173 & -0.172 & -0.172 & -0.172 & -0.172 & -0.172 \\
& $(0.110)$ & $(0.132)$ & $(0.129)$ & $(0.127)$ & $(0.128)$ & $(0.134)$ \\
\hline$N$ & 181 & 181 & 181 & 181 & 181 & 181 \\
AIC & -9.59 & -12.27 & -12.51 & -10.65 & -10.59 & -8.67 \\
BIC & 3.21 & 3.72 & 0.29 & 5.34 & 5.40 & 10.52 \\
RMSE & 0.230 & 0.227 & 0.228 & 0.228 & 0.228 & 0.228 \\
\hline
\end{tabular}

Note: Standard errors in parentheses; ${ }^{*} p<0.05,{ }^{* *} p<0.01,{ }^{* * *} p<0.001$.

series, several $\operatorname{ARMA}(p, q)$ models of the form

$$
M E G_{t}=a+\sum_{j=1}^{p} \phi_{j} M E G_{t-j}+\varepsilon_{t}+\sum_{j=1}^{q} \theta_{j} \varepsilon_{t-j}
$$

where $\varepsilon_{t}$ is assumed to be an i.i.d. white noise process, and $\phi$ and $\theta$ are the vectors of autoregressive and moving average coefficients, respectively. Table 6 presents the estimates along with goodness-offit statistics AIC and BIC. ${ }^{18}$ Based on the AIC criterion, the best model specification is an $\operatorname{ARMA}(1,1)$, but the BIC criterion chooses the $\operatorname{ARMA}(2,2)$. The predictions obtained from these models, shown in Figure 11, are very close to each other. In terms of their predictive accuracy, all models are practically identical, with a RMSE approximately equal to 0.23 .

\footnotetext{
${ }^{18}$ Several alternative $\operatorname{ARMA}(p, q)$ models were estimated; Table 6 reports those with the smallest AIC and BIC statistics
} 
Figure $11 \operatorname{ARMA}(1,1)$ and $\operatorname{ARMA}(2,2)$ Models. Observed and Predicted Values

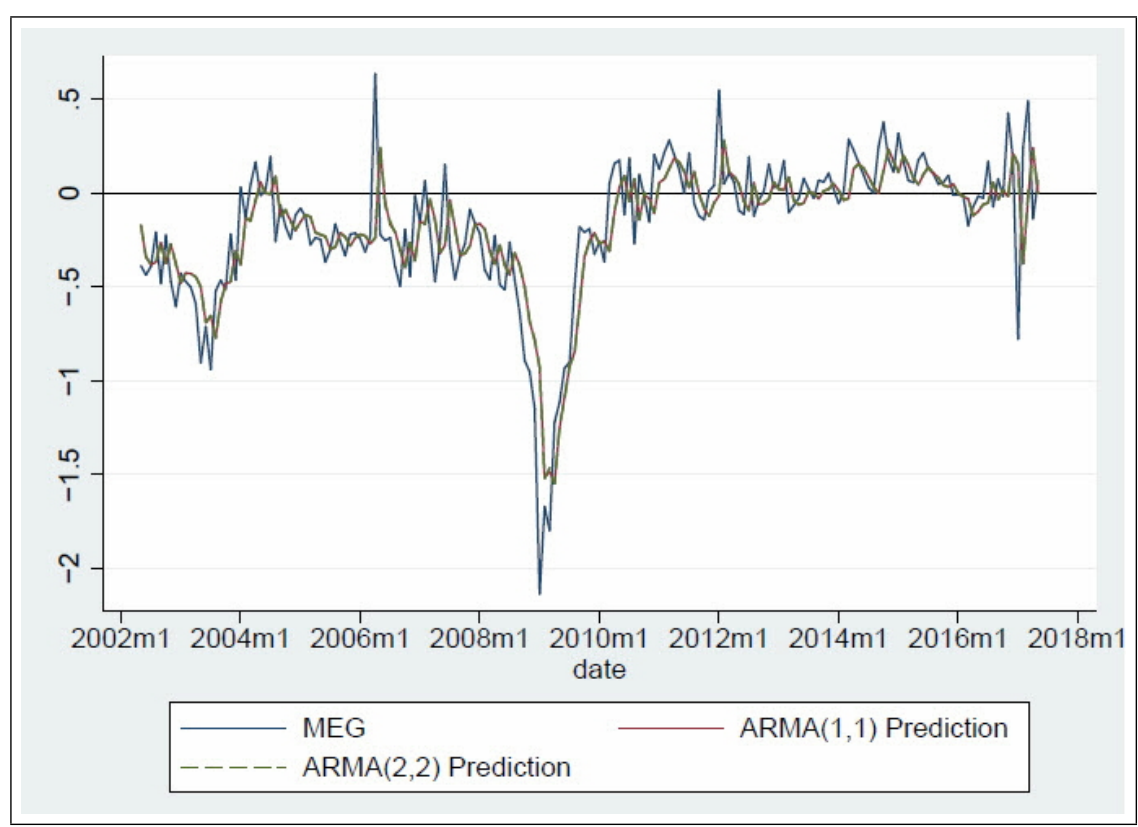

\section{Linear models of MEG}

We now incorporate the diffusion indices calculated from the FRBR surveys to assess how well they explain economic changes in the Fifth District. We proceed by estimating several linear models of MEG using contemporaneous and lagged values of the FRBR survey series. These models are generally described by the expression

$$
M E G_{t}=\alpha+\sum_{j=0}^{3} \beta_{j} X_{t-j}+\sum_{j=1}^{3} \gamma_{j} M E G_{t-j}+\varepsilon_{t},
$$

where $X_{t}$ is a vector of diffusion indices produced by the FRBR, and $\varepsilon_{t}$ is an error term assumed to be an i.i.d. white noise process.

Table 7 presents the parameter estimates of five alternative model specifications that only include contemporaneous values of the FRBR series as explanatory variables. ${ }^{19}$ In other words, those models assume

\footnotetext{
${ }^{19}$ In this section, we use the normalization $w^{u}=w^{d}=1$ and $w^{s}=0$. This means that when the percentage of participants reporting an increase is equal to the percentage
} 
Table 7 Linear Models of MEG: Contemporaneous Regressors

\begin{tabular}{|c|c|c|c|c|c|}
\hline$M E G_{t}$ & (1) & $(2)$ & $(3)$ & (4) & $(5)$ \\
\hline$R I C_{t}$ & $\begin{array}{l}0.022^{* * *} \\
(0.002)\end{array}$ & & & & \\
\hline$R I C_{t}^{E}$ & & $\begin{array}{l}0.030 * * * \\
(0.002)\end{array}$ & $\begin{array}{l}0.027^{* * *} \\
(0.003)\end{array}$ & $\begin{array}{l}0.018^{* * *} \\
(0.003)\end{array}$ & $\begin{array}{l}0.018^{* * *} \\
(0.003)\end{array}$ \\
\hline$R I C_{t}^{O}$ & & & $\begin{array}{l}-0.003 \\
(0.004)\end{array}$ & $\begin{array}{l}-0.002 \\
(0.005)\end{array}$ & \\
\hline$R I C_{t}^{S}$ & & & $\begin{array}{l}0.007 \\
(0.004)\end{array}$ & $\begin{array}{l}0.007 \\
(0.004)\end{array}$ & $\begin{array}{l}0.006 \\
(0.003)\end{array}$ \\
\hline$R I C_{t}^{B}$ & & & & $\begin{array}{l}0.001 \\
(0.004)\end{array}$ & \\
\hline$R I C_{t}^{C}$ & & & & $\begin{array}{c}-0.010^{*} \\
(0.004)\end{array}$ & $\begin{array}{l}-0.010^{* *} \\
(0.004)\end{array}$ \\
\hline$R I C_{t}^{V}$ & & & & $\begin{array}{l}0.006 \\
(0.004)\end{array}$ & $\begin{array}{l}0.006 \\
(0.004)\end{array}$ \\
\hline$R I C_{t}^{H}$ & & & & $\begin{array}{l}0.006 \\
(0.004)\end{array}$ & $\begin{array}{l}0.005 \\
(0.004)\end{array}$ \\
\hline$R I C_{t}^{W}$ & & & & $\begin{array}{l}0.011^{*} \\
(0.005)\end{array}$ & $\begin{array}{l}0.011^{*} \\
(0.004)\end{array}$ \\
\hline$R I C_{t}^{I F}$ & & & & $\begin{array}{l}-0.012^{* * *} \\
(0.003)\end{array}$ & $\begin{array}{l}-0.012^{* * *} \\
(0.003)\end{array}$ \\
\hline$R I C_{t}^{I R}$ & & & & $\begin{array}{l}0.005 \\
(0.004)\end{array}$ & $\begin{array}{l}0.005 \\
(0.004)\end{array}$ \\
\hline Constant & $\begin{array}{l}-0.186 \text { *** } \\
(0.022)\end{array}$ & $\begin{array}{l}-0.160 \text { *** } \\
(0.019)\end{array}$ & $\begin{array}{l}-0.170 \text { *** } \\
(0.019)\end{array}$ & $\begin{array}{c}-0.167 \\
(0.090)\end{array}$ & $\begin{array}{c}-0.179^{*} \\
(0.075)\end{array}$ \\
\hline$N$ & 181 & 181 & 181 & 181 & 181 \\
\hline $\operatorname{Adj}-R^{2}$ & 0.429 & 0.578 & 0.587 & 0.640 & 0.644 \\
\hline RMSE & 0.292 & 0.251 & 0.248 & 0.232 & 0.231 \\
\hline
\end{tabular}

Note: Newey-West standard errors in parentheses; ${ }^{*} p<0.05,{ }^{* *} p<0.01,{ }^{* * *} p<$ 0.001 .

$\beta_{j}=\gamma_{j}=0$, for $j=1,2,3$. Model (1) only includes the FRBR composite diffusion index, i.e., $X_{t}=R I C_{t}$; in model (2), $X_{t}=R I C_{t}^{E}$, which is the FRBR diffusion index that tracks changes in employment; model (3) includes the components of the FRBR composite index, i.e., $X_{t}$ $=\left[R I C_{t}^{E}, R I C_{t}^{S}, R I C_{t}^{O}\right]$; model (4) incorporates additional diffusion

of participants reporting a decrease, the diffusion index is equal to zero. We use this normalization to avoid working with small numbers with many digits. Ideally, we would want to rescale the ISM diffusion index. However, this requires using the ISM raw data, which are not publicly available. 
Figure 12 Observed and Predicted Values of MEG: Model (5) (Stepwise Selection)

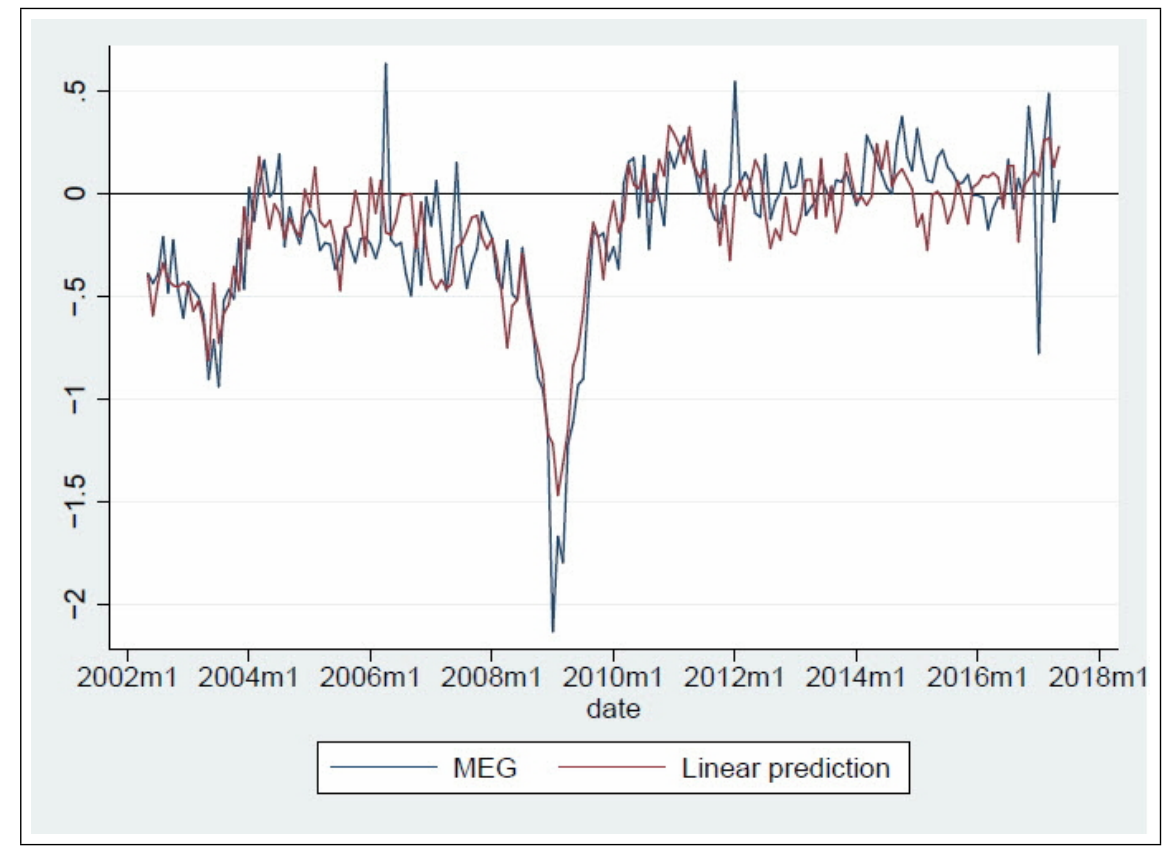

indices from the FRBR surveys; and model (5) is a refinement of model (4) obtained after a stepwise procedure of regressor selection. ${ }^{20}$

A few remarks are worth making. First, by inspecting model (1), it follows that when the composite diffusion index $R I C_{t}$ is equal to zero, $M E G_{t}=-0.186$. In other words, zero employment growth in the district would be consistent with a value of $R I C_{t}=8.5$. It is important to note that, in theory, a zero diffusion index does not imply a zero growth rate. A diffusion index captures the breadth of a change measured by the number of respondents experiencing no change, an increase, or a decrease in a specific variable. In other words, a diffusion index tracks changes in the extensive margin. A growth rate, however, in addition

\footnotetext{
${ }^{20}$ Standard errors are produced by a Newey-West regression procedure that corrects potential serial correlation in the error terms. While under serial correlation, OLS still produces unbiased parameter estimates; the standard errors in this case are not efficient. We reestimate the model in (5) using the Newey-West regression procedure that produces serial correlation robust standard errors. The adjusted- $R^{2}$ measure is from the OLS regression.
} 
to changes in the extensive margin, also captures the intensity of the change, or the intensive margin. Pinto et al. (2015a) and Pinto et al. (2015b) show a decomposition of a growth rate into the extensive margin, or a term that includes a diffusion index, and the intensive margin. So the explanatory power of the diffusion indices depends both on the information content of the FRBR surveys, summarized by the diffusion indices, and, more generally, on the extent to which changes in the extensive margin drive changes in the growth rate.

Second, the employment diffusion index $R I C_{t}^{E}$ by itself (model [2]) explains about 60 percent of the variation in MEG. In this case, when $R I C_{t}^{E}=5.33, M E G_{t}=0$. Since information about $R I C_{t}^{E}$ is available prior to the release of the $M E G_{t}$ monthly data (usually, the value of $R I C_{t}^{E}$ is known a few weeks earlier), it becomes important to understand such a relationship in order to anticipate the values of $M E G_{t}$. Third, adding more information from the FRBR surveys improves the fit of the model, as shown by models (4) and (5). In those cases, the employment diffusion index $R I C_{t}^{E}$ is still the most important variable explaining the behavior of $M E G_{t}$. Other variables, such as $R I C_{t}^{C}$, $R I C_{t}^{W}$, and $R I C_{t}^{I F}$, also contribute to explaining $M E G_{t}$. Note that all the models considered in Table 7 have a relatively low adjusted- $R^{2}$; model (5) has the highest one, which is equal to 0.644. Figure 12 plots the observed and predicted values of this model. It shows that the two lines only infrequently overlap, confirming the model's low goodness of fit. This suggests, in light of the previous discussion on growth rates and extensive and intensive margins, that in the case of Fifth District employment, diffusion indices (or the extensive margin) only partially explain its growth rate. In other words, a low adjusted- $R^{2}$ should not be necessarily used to draw conclusions about the quality of the information content of the FRBR surveys. In fact, Pinto et al. (2015b) show that $R I C_{t}^{E}$ tracks fairly well the extensive margin component of the actual employment growth rate in the Fifth District.

Next, we perform a similar exercise as in Section 2.2 but for the MEG series. In this case, we obtain the following results: $\alpha=-0.1$, $\beta^{E}=0.83, \beta^{O}=0.00, \beta^{S}=0.17$. The main conclusion from this exercise is that a composite index that assigns weights to the individual diffusion indices $\left\{R I C_{t}^{S}, R I C_{t}^{S}, R I C_{t}^{O}\right\}$ with the objective of tracking $M E G_{t}$ as closely as possible should give the highest weight to $R I C_{t}^{E}$, a lower but positive weight to $R I C_{t}^{O}$, and zero weight to $R I C_{t}^{O}$. This composite index is definitely different from the one that is supposed to track the national economy and is also different from the one currently reported by the FRBR.

Up to this point, the models assume a contemporaneous relationship between the variables. The models examined next, summarized in 
Table 8 Linear Models of MEG: Contemporaneous and Lagged Regressors

\begin{tabular}{|c|c|c|c|c|c|c|}
\hline$M E G_{t}$ & (1) & (2) & (3) & (4) & (5) & (6) \\
\hline \multirow[t]{2}{*}{$M E G_{t-1}$} & & $0.315^{* * *}$ & & $0.320^{* * *}$ & & $0.278^{* * *}$ \\
\hline & & $(0.075)$ & & $(0.078)$ & & $(0.079)$ \\
\hline \multirow[t]{2}{*}{$M E G_{t-2}$} & & 0.097 & & 0.060 & & 0.055 \\
\hline & & $(0.079)$ & & $(0.083)$ & & $(0.082)$ \\
\hline \multirow[t]{2}{*}{$M E G_{t-3}$} & & $0.182^{*}$ & & 0.081 & & 0.129 \\
\hline & & $(0.071)$ & & $(0.075)$ & & $(0.079)$ \\
\hline$R^{2} C_{t}$ & $\begin{array}{c}0.010^{* * *} \\
(0.002)\end{array}$ & $\begin{array}{c}0.008^{* * *} \\
(0.002)\end{array}$ & & & & \\
\hline \multirow[t]{2}{*}{$R I C_{t-1}$} & $0.006^{*}$ & 0.003 & & & & \\
\hline & $(0.003)$ & $(0.002)$ & & & & \\
\hline \multirow[t]{2}{*}{$R I C_{t-2}$} & 0.004 & -0.000 & & & & \\
\hline & $(0.003)$ & $(0.002)$ & & & & \\
\hline$R I C_{t-3}$ & $\begin{array}{c}0.011^{* * *} \\
(0.002)\end{array}$ & $\begin{array}{c}0.003 \\
(0.002)\end{array}$ & & & & \\
\hline \multirow[t]{2}{*}{$R I C_{t}^{E}$} & & & $0.015^{* * *}$ & $0.010^{* * *}$ & $0.010^{* *}$ & 0.005 \\
\hline & & & $(0.003)$ & $(0.003)$ & $(0.003)$ & $(0.003)$ \\
\hline \multirow[t]{2}{*}{$R I C_{t-1}^{E}$} & & & $0.010^{* *}$ & $0.007^{*}$ & $0.008^{*}$ & 0.005 \\
\hline & & & $(0.003)$ & (0.003) & $(0.004)$ & $(0.003)$ \\
\hline \multirow{2}{*}{$R I C_{t-2}^{E}$} & & & 0.002 & -0.002 & 0.003 & -0.001 \\
\hline & & & $(0.003)$ & (0.003) & $(0.004)$ & $(0.003)$ \\
\hline \multirow{2}{*}{$R I C_{t-3}^{E}$} & & & $0.008^{* *}$ & 0.004 & $0.008^{*}$ & 0.004 \\
\hline & & & $(0.003)$ & $(0.003)$ & $(0.003)$ & $(0.003)$ \\
\hline $\mathrm{RIC}_{t}^{S}$ & & & & & $\begin{array}{c}0.003 \\
(0.003)\end{array}$ & $\begin{array}{c}0.002 \\
(0.003)\end{array}$ \\
\hline \multirow{2}{*}{$R I C_{t-1}^{S}$} & & & & & 0.004 & 0.003 \\
\hline & & & & & $(0.003)$ & $(0.003)$ \\
\hline \multirow[t]{2}{*}{$R I C_{t-2}^{S}$} & & & & & 0.004 & 0.002 \\
\hline & & & & & $(0.003)$ & $(0.003)$ \\
\hline \multirow{2}{*}{$\operatorname{RIC}_{t-3}^{S}$} & & & & & 0.002 & -0.001 \\
\hline & & & & & $(0.003)$ & $(0.003)$ \\
\hline \multirow[t]{2}{*}{$\operatorname{RIC}_{t}^{U}$} & & & & & 0.002 & 0.003 \\
\hline & & & & & $(0.004)$ & $(0.003)$ \\
\hline \multirow[t]{2}{*}{$R I C_{t-1}^{O}$} & & & & & -0.003 & -0.001 \\
\hline & & & & & $(0.004)$ & $(0.003)$ \\
\hline \multirow[t]{2}{*}{$R I C_{t-2}^{O}$} & & & & & -0.005 & -0.002 \\
\hline & & & & & $(0.004)$ & $(0.003)$ \\
\hline \multirow[t]{2}{*}{$R I C_{t-3}^{O}$} & & & & & -0.000 & 0.002 \\
\hline & & & & & $(0.004)$ & $(0.003)$ \\
\hline \multirow[t]{2}{*}{ Constant } & $-0.186^{* * *}$ & $-0.075^{* * *}$ & $-0.155^{* * *}$ & $-0.082^{* * *}$ & $-0.174^{* * *}$ & $-0.093^{* * *}$ \\
\hline & $(0.019)$ & $(0.020)$ & $(0.017)$ & $(0.022)$ & $(0.018)$ & $(0.024)$ \\
\hline$N$ & 178 & 178 & 178 & 178 & 178 & 178 \\
\hline Adj. $R^{2}$ & 0.596 & 0.713 & 0.660 & 0.702 & 0.673 & 0.709 \\
\hline RMSE & 0.247 & 0.208 & 0.227 & 0.212 & 0.222 & 0.209 \\
\hline
\end{tabular}

Note: Newey-West standard errors in parentheses; ${ }^{*} p<0.05,{ }^{* *} p<0.01,{ }^{* * *} p<$ 0.001 .

Table 8, include both contemporaneous and lagged regressors, as specified in expression (11). The results show that by considering a dynamic relationship between the variables, it is possible to improve the fit of the models. Lagged values of $R I C_{t}^{E}$ are relevant for explaining the behavior of $M E G_{t}$ when $R I C_{t}^{E}$ is the only explanatory variable (model 
[3]), when $R I C_{t}^{E}$ is combined with $M E G_{t}$ (model [4]), and when $R I C_{t}^{E}$ is included in the regression model along with $R I C_{t}^{S}$ and $R I C_{t}^{O}$. Note, however, that $R I C_{t}^{E}$ becomes statistically insignificant when all individual diffusion indices and their lags, in addition to lagged values of $M E G_{t}$, are included in the model specification (model [6]). The latter result is consistent with the persistent behavior of the $M E G_{t}$ series described earlier (shown in Figure 4), and the fact that the series $R I C_{t}^{E}$, $R I C_{t}^{S}$, and $R I C_{t}^{O}$ are highly correlated. In sum, all the models that include lagged values of $M E G_{t}$ (specifically, models [2], [4], and [6]) have relatively low RMSEs. However, the lowest RMSE (and also the highest adjusted- $R^{2}$ ) is associated with model (2), with a RMSE equal to 0.208 .

Finally, Table 9 presents the best dynamic specification that includes all the diffusion indices calculated by the FRBR. We report the estimates for the model that results from a stepwise variable selection process. Of all the models considered up to this point, this last specification has the highest predictive accuracy with a RMSE of 0.189. In addition to the lagged values of $M E G_{t}$, a number of diffusion indices not currently included in the reported composite index, specifically $R I C_{t}^{I R}$ and $R I C_{t}^{I F}$, appear to be significantly different from zero. The model has, however, an adjusted- $R^{2}$ equal to 0.76 , so it imperfectly fits the data. Figure 13 shows observed and predicted values from this specification.

\section{VAR Models}

\section{Bivariate VAR model: $M E G$ and FRBR composite diffusion index}

As in the ISM case, we estimate several VAR models, assess their predictive accuracy, and perform a FEVD. We begin by estimating two bivariate VAR models: one includes the FRBR composite index $R I C_{t}$ and the other the FRBR employment index $R I C_{t}^{E}$. The results of the estimation are shown in Tables 21 and 22 in Appendix A.4.1 in addition to the observed and predicted values obtained from each model (Figures 20 and 21, respectively). Comparing the accuracy of the predictions, the specification that uses the individual diffusion index $R I C_{t}^{E}$ has a RMSE equal to 0.211 , slightly below the model that includes the composite diffusion index $R I C_{t}$, with a RMSE equal to 0.217 , and lower AIC and BIC statistics. Note, however, that some of the models considered in the previous section outperform, in terms of predictive accuracy, these two VAR models. Finally, the FEVDs for each model, shown in Figures 14a and 14b, are practically identical. They indicate 
Table 9 Linear Model of MEG: Stepwise Selection

\begin{tabular}{|c|c|}
\hline & $M E G_{f}$ \\
\hline$R_{\text {RIC }}^{E}$ & $\begin{array}{c}0.006 \\
(0.004)\end{array}$ \\
\hline$R I C_{t-1}^{E}$ & $\begin{array}{c}0.003 \\
(0.003)\end{array}$ \\
\hline$R I C_{t-3}^{E}$ & $\begin{array}{c}0.003 \\
(0.003)\end{array}$ \\
\hline$R_{1} C_{t-2}^{O}$ & $\begin{array}{c}0.002 \\
(0.002)\end{array}$ \\
\hline$R I C_{t-3}^{O}$ & $\begin{array}{c}0.006 \\
(0.003)\end{array}$ \\
\hline$R_{\text {RIC }}^{S}$ & $\begin{array}{c}0.005 \\
(0.003)\end{array}$ \\
\hline$R I C_{t-1}^{S}$ & $\begin{array}{c}0.004 \\
(0.003)\end{array}$ \\
\hline$R I C_{t-3}^{S}$ & $\begin{array}{l}-0.003 \\
(0.003)\end{array}$ \\
\hline$\overline{R I C_{t}^{C}}$ & $\begin{array}{l}-0.003 \\
(0.003)\end{array}$ \\
\hline$R I C_{t-1}^{C}$ & $\begin{array}{l}-0.006 \\
(0.003)\end{array}$ \\
\hline$R I C_{t-1}^{V}$ & $\begin{array}{c}0.004 \\
(0.004)\end{array}$ \\
\hline$R I C_{t-2}^{V}$ & $\begin{array}{l}-0.006 \\
(0.004)\end{array}$ \\
\hline $\mathrm{RIC}_{t}^{W}$ & $\begin{array}{c}0.005 \\
(0.004)\end{array}$ \\
\hline$R I C_{t-1}^{W}$ & $\begin{array}{c}0.005 \\
(0.004)\end{array}$ \\
\hline$R I C_{t-2}^{W}$ & $\begin{array}{l}-0.008 \\
(0.004)\end{array}$ \\
\hline $\mathrm{RIC}_{t}^{H}$ & $\begin{array}{c}0.004 \\
(0.003)\end{array}$ \\
\hline$R I C_{t-1}^{H}$ & $\begin{array}{l}-0.002 \\
(0.003)\end{array}$ \\
\hline$R I C_{t-3}^{H}$ & $\begin{array}{c}0.002 \\
(0.003)\end{array}$ \\
\hline$R I C_{t-1}^{I R}$ & $\begin{array}{l}-0.010^{*} \\
(0.004)\end{array}$ \\
\hline$R I C_{t-2}^{I R}$ & $\begin{array}{c}0.004 \\
(0.004)\end{array}$ \\
\hline$R I C_{t-3}^{I R}$ & $\begin{array}{r}0.013^{* * *} \\
(0.004)\end{array}$ \\
\hline$R I C_{t-1}^{I F}$ & $\begin{array}{c}0.006 \\
(0.004)\end{array}$ \\
\hline$R I C_{t-2}^{I F}$ & $\begin{array}{r}-0.012^{* *} \\
(0.004)\end{array}$ \\
\hline$M E G_{t-1}$ & $\begin{array}{r}0.249^{* * *} \\
(0.072)\end{array}$ \\
\hline$M E G_{t-3}$ & $\begin{array}{l}0.155^{*} \\
(0.070)\end{array}$ \\
\hline Constant & $\begin{array}{c}-0.140 \\
(0.081) \\
\end{array}$ \\
\hline $\mathrm{N}$ & 178 \\
\hline Adjusted $R^{2}$ & 0.762 \\
\hline RMSE & 0.189 \\
\hline
\end{tabular}

Note: Newey-West standard errors in parentheses; ${ }^{*} p<0.05,{ }^{* *} p<0.01,{ }^{* * *} p<$ 0.001 . 
Figure 13 Dynamic Linear Model of MEG: Stepwise Selection

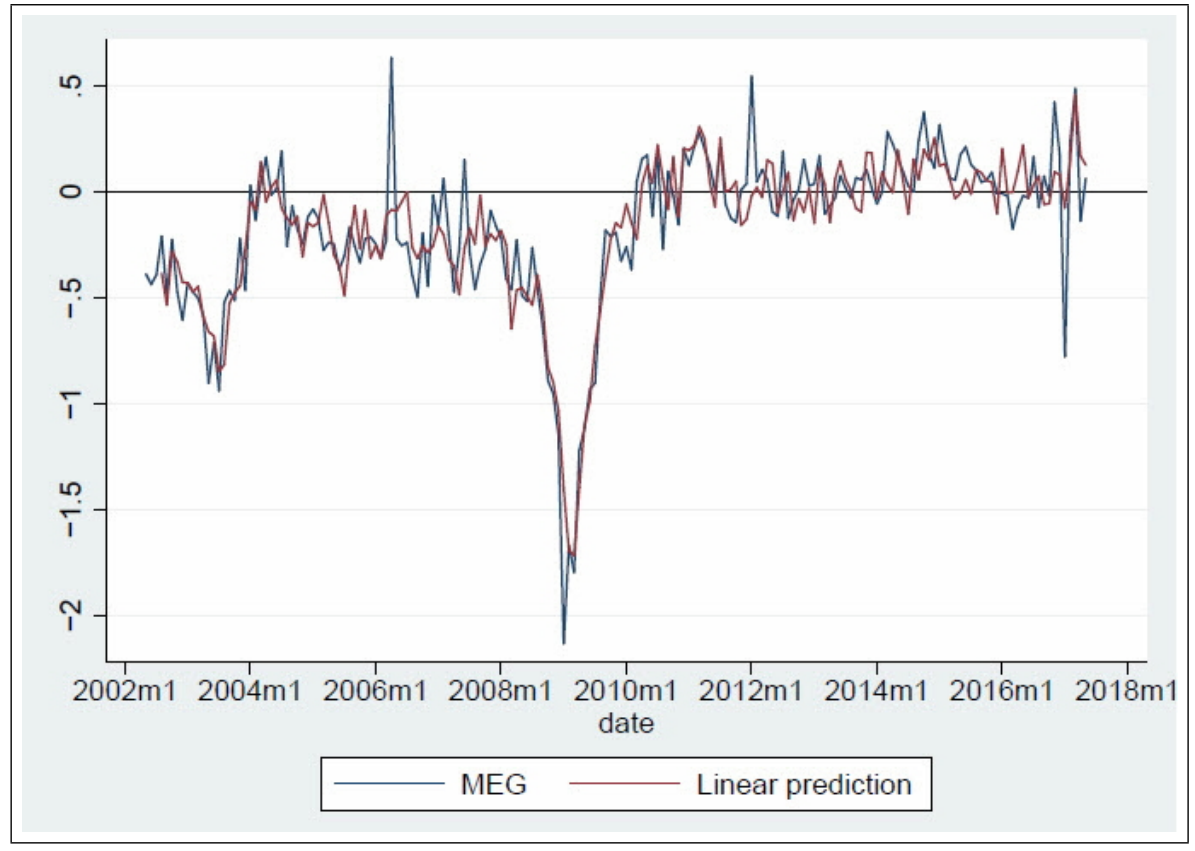

that, even though MEG explains most of the variation in the series, the FRBR diffusion indices are still relevant: $R I C_{t}$ and $R I C_{t}^{E}$ explain, in each case, about 20 percent of the variation of MEG after eight periods.

Multivariate VAR model: MEG and FRBR individual diffusion indices

Finally, we estimate a VAR model that includes additional diffusion indices computed from the FRBR survey. We follow a stepwise regression procedure to select the components considered in the analysis. The estimated values are shown in Table 23, and the predicted values from the structural equation are presented in Figure 22 in Appendix A.4.2. This model has the highest predictive accuracy of all the models considered thus far, with a RMSE of 0.131. Of all the FRBR series included in the model, $R I C_{t}^{E}$ is still the one that explains a larger proportion of the variation in $M E G_{t}$ (around 15 percent of the variance), as shown by the FEVD in Figure 15. 
Figure 14 Forecast Error Variance Decomposition

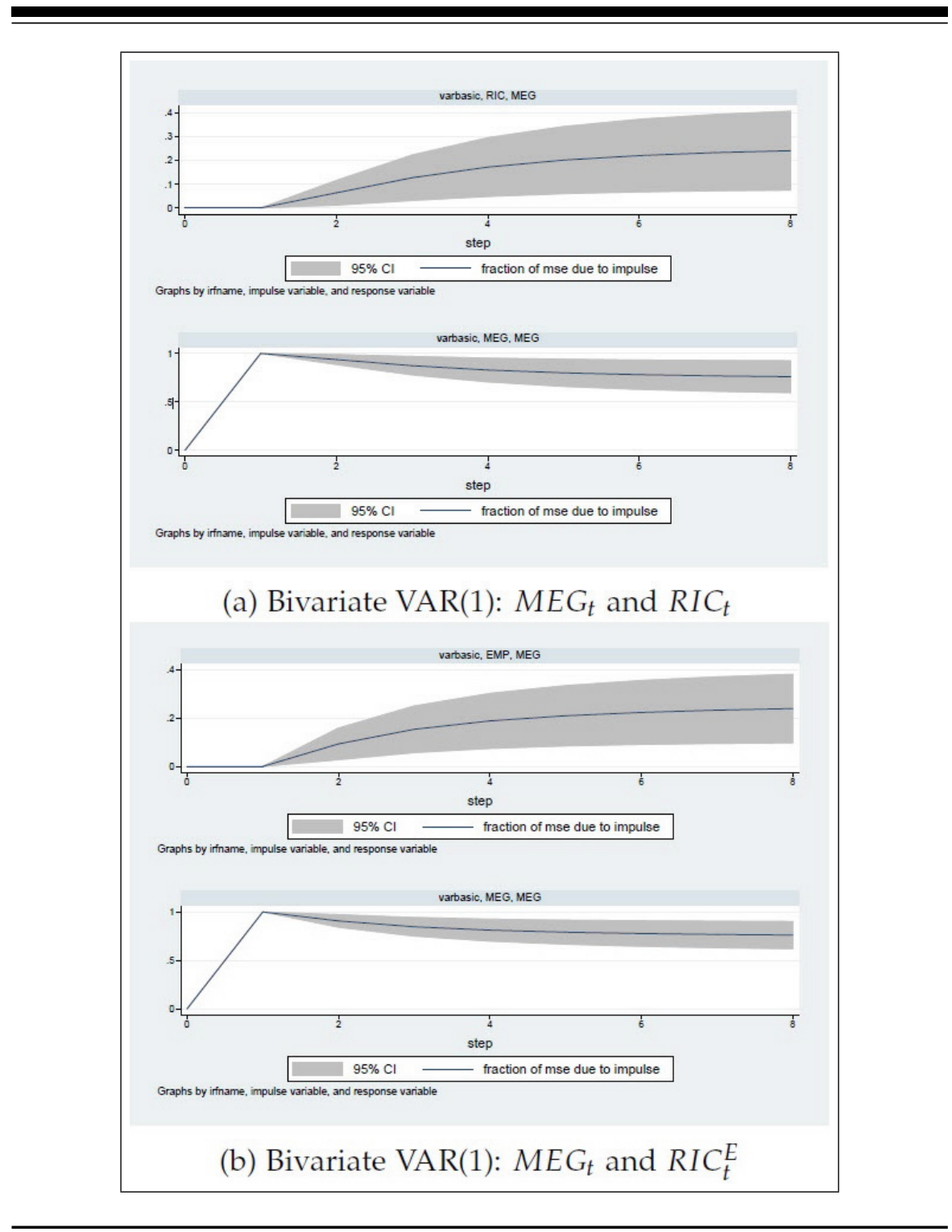

\section{Summary of results}

From all the models considered in the previous sections, we present those with the highest predictive accuracy in Table 10, in addition to the model that includes the composite index $R I C_{t}$ currently reported by the FRBR, for comparison. The VAR model that includes all the FRBR individual diffusion indices has the lowest RMSE. This model, 
Figure $15 M E G_{t}$ and FRBR Individual Diffusion Indices. Forecast Error Variance Decomposition

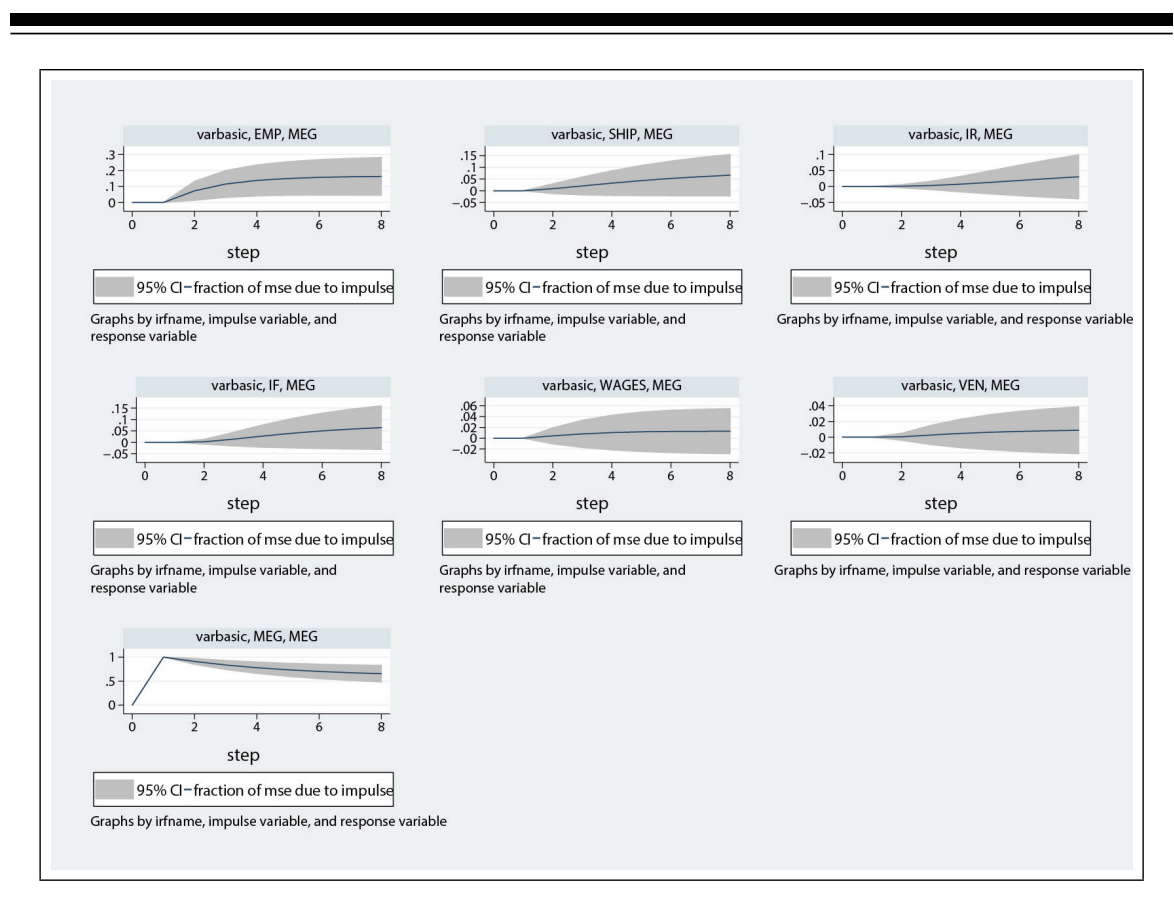

Table 10 Comparison of RMSE for Selected Models of MEG

\begin{tabular}{|c|c|c|}
\hline & & RMSE \\
\hline Composite index & $R I C_{t}$ & 0.29 \\
\hline Univariate & $\begin{array}{l}A R M A(1,1) \\
A R M A(2,2)\end{array}$ & $\begin{array}{l}0.23 \\
0.23\end{array}$ \\
\hline Linear & $\begin{array}{l}\text { Contemporaneous } \\
\text { Dynamic }\end{array}$ & $\begin{array}{l}0.23 \\
0.19\end{array}$ \\
\hline VAR & $\begin{array}{l}\text { Bivariate } R I C_{t} \\
\text { Bivariate } R_{t}^{E} \\
\text { Multivariate }\end{array}$ & $\begin{array}{l}0.22 \\
0.21 \\
0.13\end{array}$ \\
\hline
\end{tabular}

with an RMSE of 0.13 , is clearly an improvement compared with the model that relies only on $R I C_{t}$. All the other models, however, have approximately the same RMSEs. As in the ISM case, a linear dynamic model that includes readily available information from the FRBR survey performs reasonably well. 


\section{CONCLUSION}

In this paper, we evaluate the information content of the FRBR manufacturing survey to determine the extent to which the diffusion indices based on the survey responses contribute to explaining national and regional economic conditions. We do so by examining the predictive accuracy of a variety of models, some of which include the composite diffusion index reported by the FRBR, and some of which incorporate additional information available from the FRBR survey but not currently employed in the calculation of the composite index.

The findings of the exercise can be summarized as follows. First, the diffusion indices currently reported by the FRBR manufacturing survey perform reasonably well at explaining the national economy, described by the evolution of the ISM diffusion index, and the regional economy, described by the evolution of the MEG. Second, in order to more accurately predict the behavior of the national and regional economy, it becomes essential to consider models that account for a richer dynamic structure given the high persistence of the series under study. And third, there are grounds for improving the predictive power of the FRBR composite index, both at national and regional levels, by adjusting the weights currently used in the calculation and by including other readily available diffusion indices. However, it should be kept in mind that the composite indices that track the national and regional economy would not necessarily be the same. This paper provides a few insights on what those diffusion indices would look like.

Future analysis should study more carefully the design of composite indices based on currently available information, including perhaps the possibility of constructing those indices based on a principal component analysis. 


\section{APPENDIX A.1: UNIT ROOT TESTS}

Table 11 Unit Root Tests

\begin{tabular}{lcccccc}
\hline \hline \multirow{2}{*}{ Variable } & \multicolumn{2}{c}{ Drift } & \multicolumn{2}{c}{ Drift and Trend } & \multicolumn{2}{c}{ ADF Test } \\
& t-stat & p-value & t-stat & p-value & t-stat & p-value \\
\hline$I S M_{t}$ & -2.591 & 0.095 & -2.801 & 0.058 & -3.437 & 0.047 \\
$M E G_{t}$ & -4.632 & 0.000 & -5.199 & 0.000 & -3.122 & 0.021 \\
$R I C_{t}$ & -5.132 & 0.000 & -5.257 & 0.000 & -3.902 & 0.012 \\
$R_{t}^{E}$ & -4.557 & 0.000 & -5.130 & 0.000 & -3.461 & 0.044 \\
$R_{t}^{O}$ & -5.708 & 0.000 & -5.757 & 0.000 & -4.358 & 0.003 \\
$R_{t}^{S}$ & -6.624 & 0.000 & -6.647 & 0.000 & -4.069 & 0.007 \\
$R_{t}^{B}$ & -6.082 & 0.000 & -6.176 & 0.000 & -4.587 & 0.001 \\
$R_{t}^{C}$ & -6.088 & 0.000 & -6.134 & 0.000 & -4.326 & 0.003 \\
$R_{t}^{H}$ & -5.717 & 0.000 & -6.034 & 0.000 & -5.348 & 0.000 \\
$R_{t}^{W}$ & -6.346 & 0.000 & -6.660 & 0.000 & -3.130 & 0.099 \\
$R_{t}^{I F}$ & -4.558 & 0.000 & -4.775 & 0.000 & -3.415 & 0.049 \\
$R_{t}^{I R}$ & -4.720 & 0.000 & -5.163 & 0.000 & -3.697 & 0.023 \\
$R_{t}^{V}$ & -5.276 & 0.000 & -5.294 & 0.000 & -3.749 & 0.019 \\
\hline
\end{tabular}

Note: ADF: Augmented Dickey-Fuller. The number of $\Delta$ terms in the ADF is determined by the autoregressive order. 
APPENDIX A.2: CROSS-CORRELOGRAMS

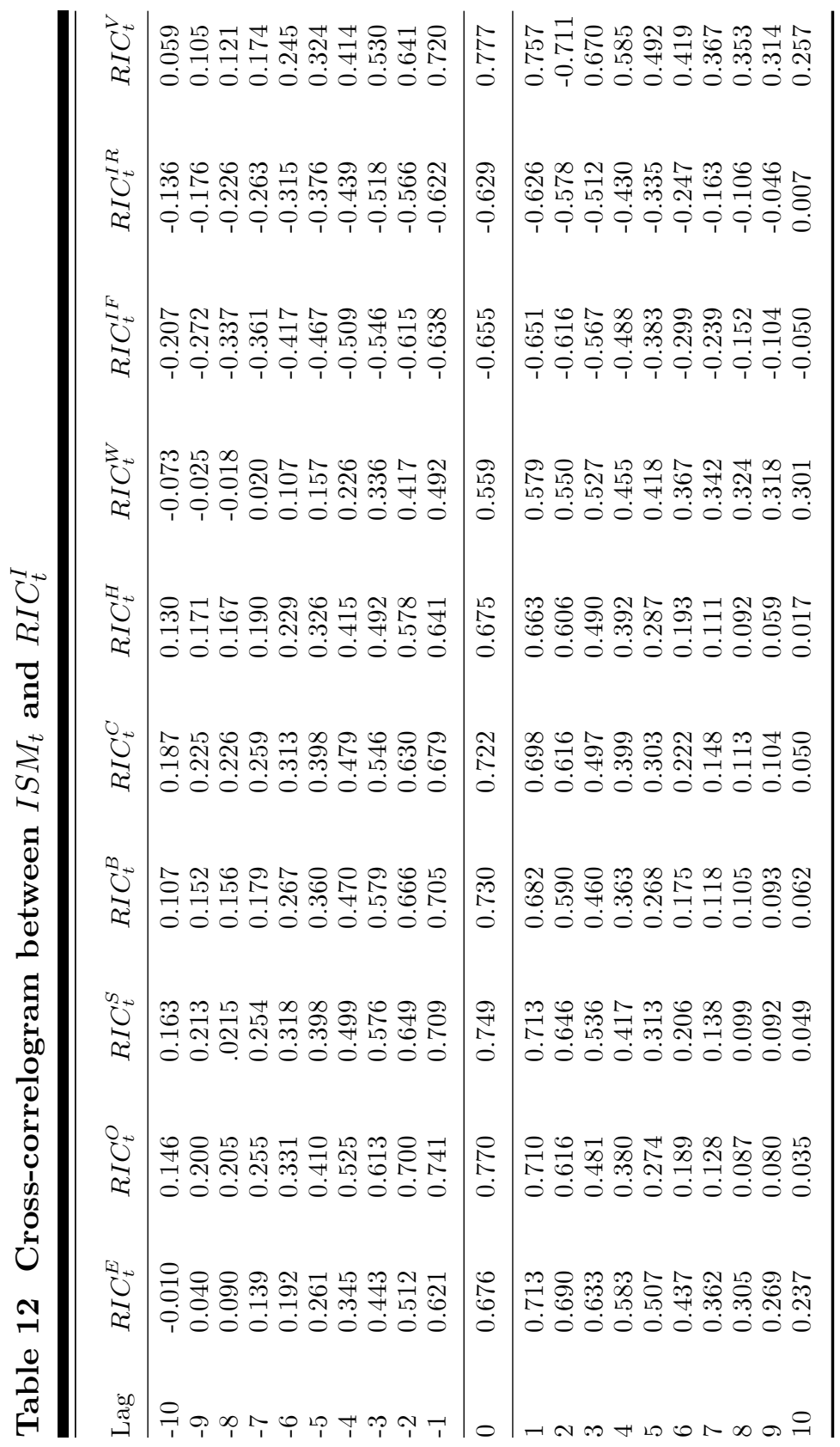




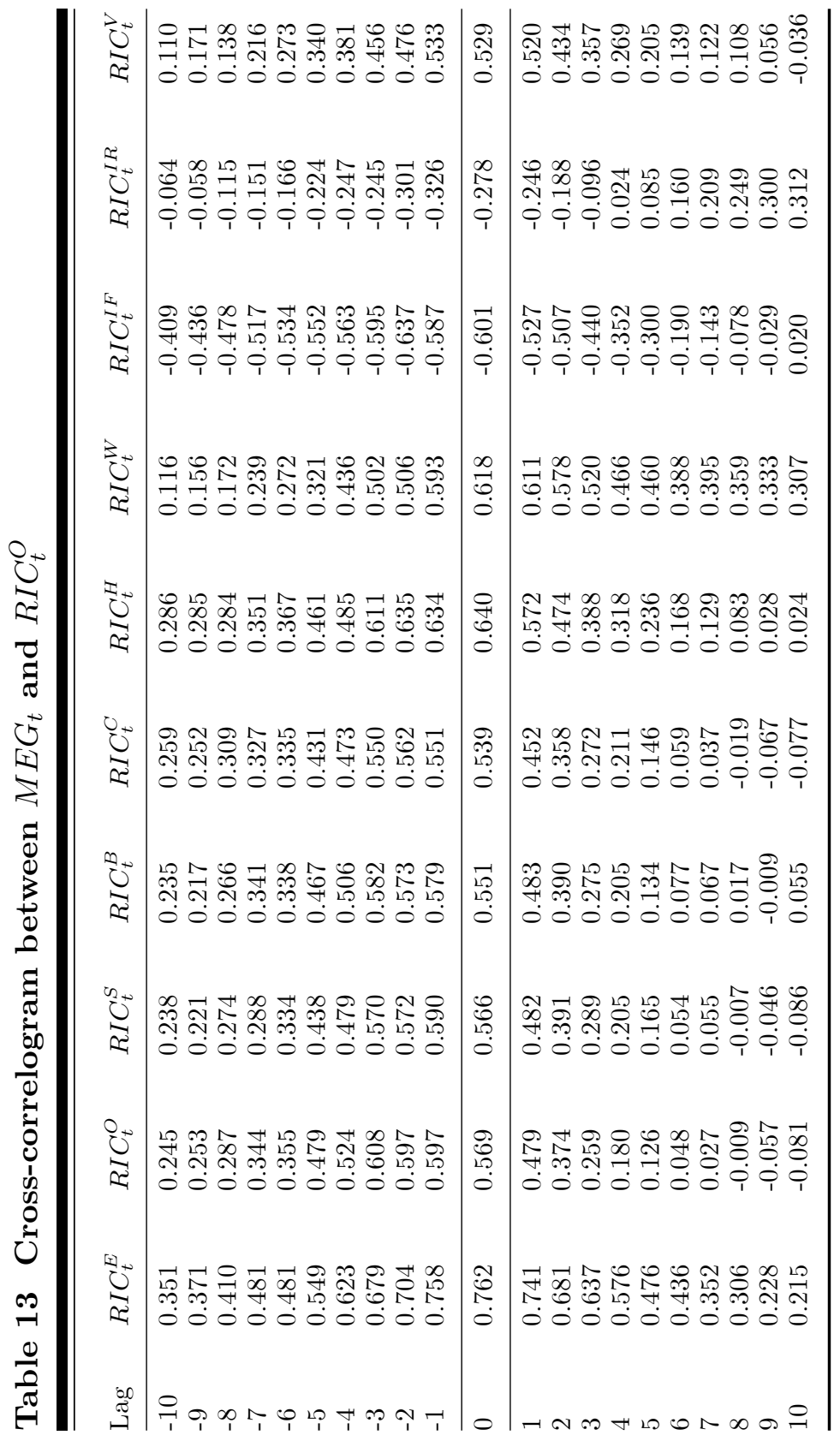




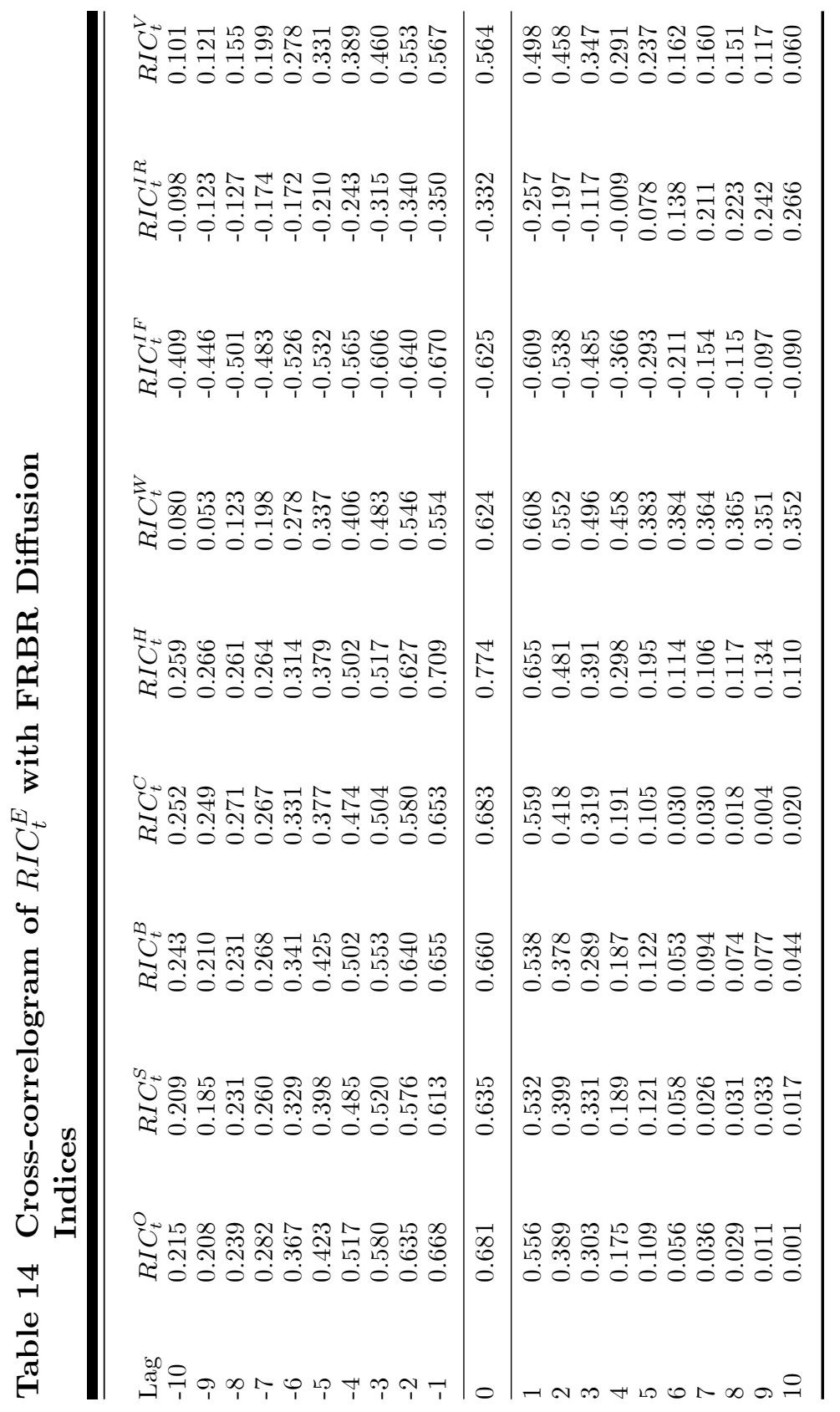




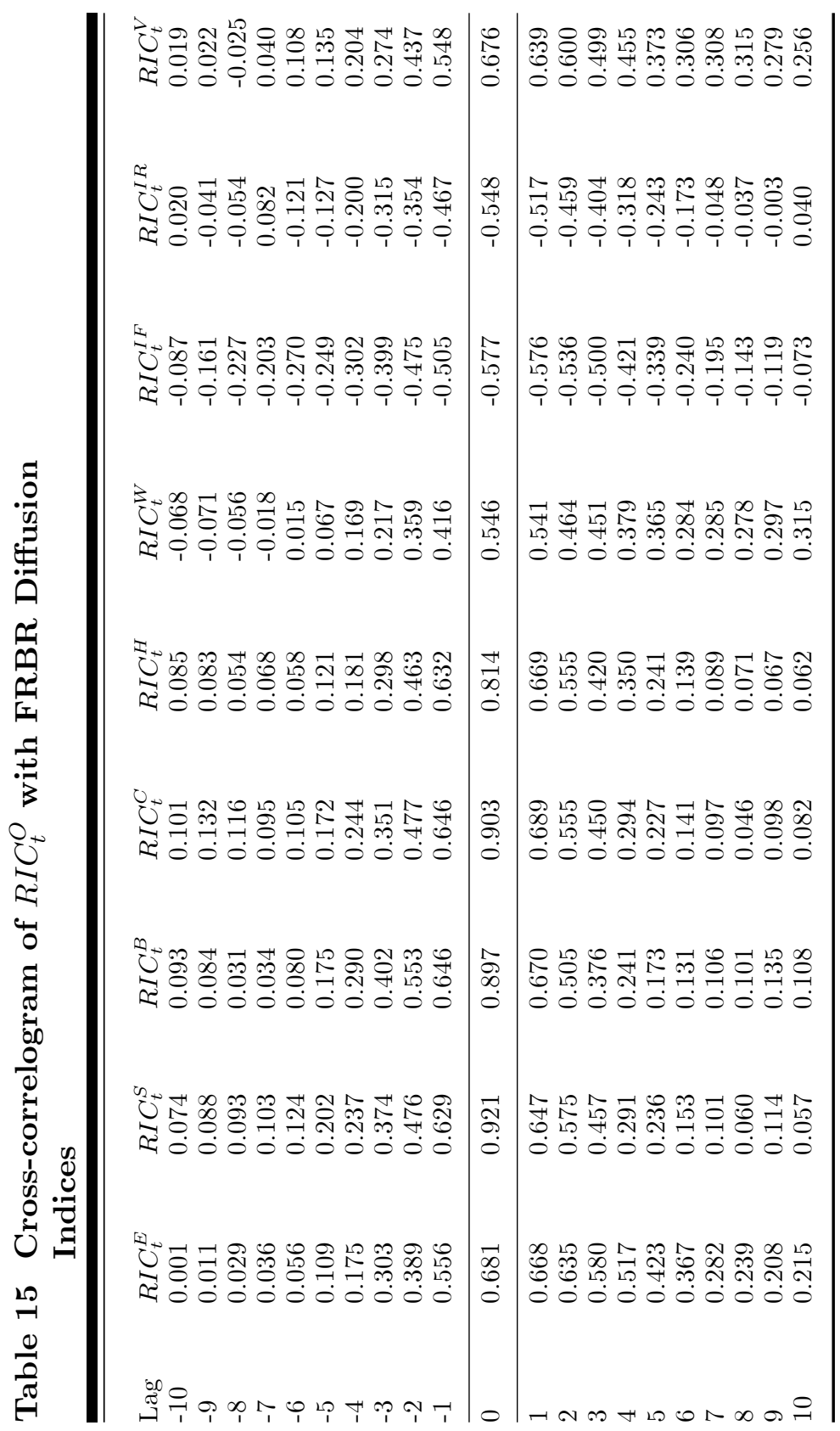




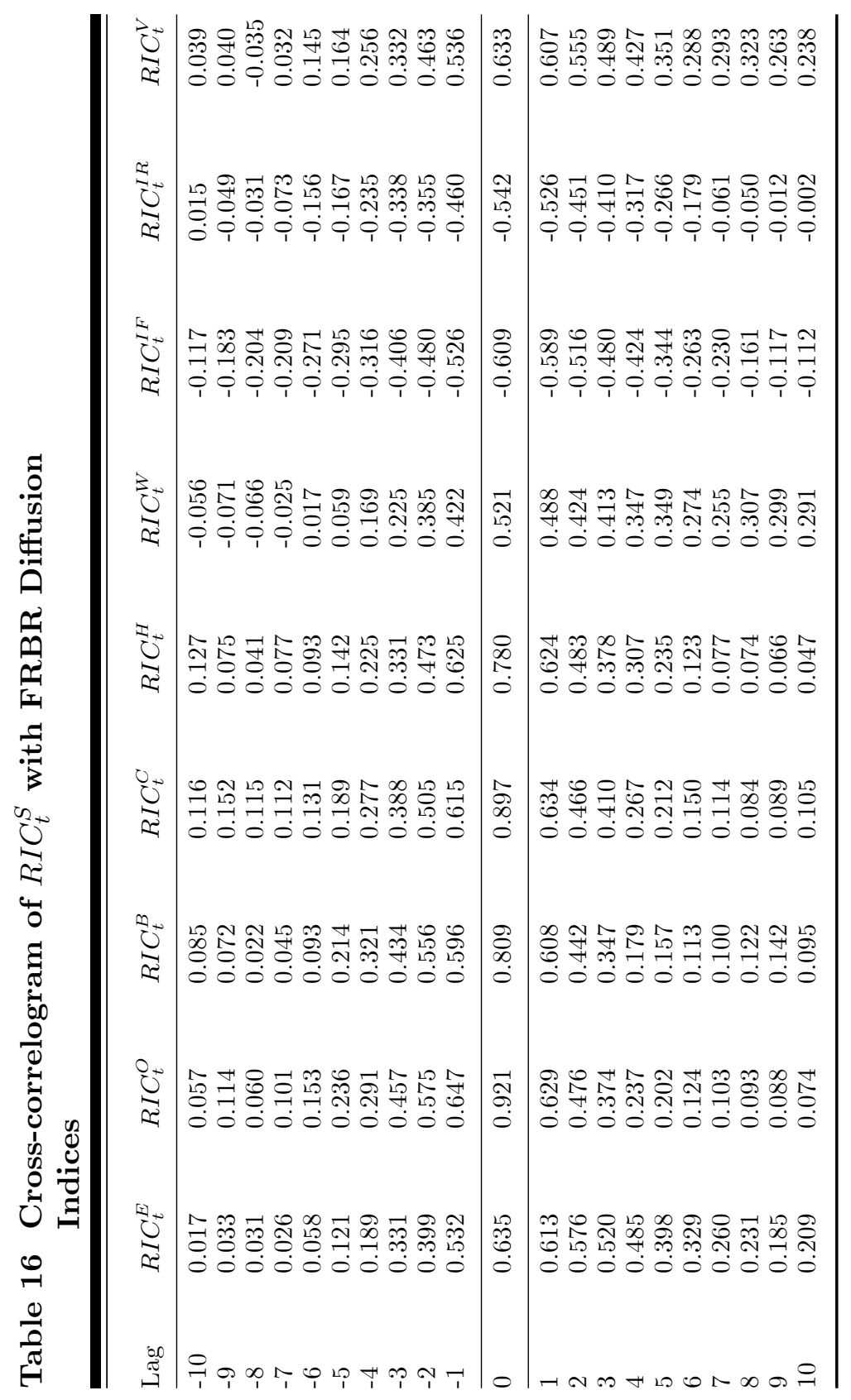


APPENDIX A.3: NATIONAL ECONOMY: VAR MODELS

\section{A.3.1 UNIVARIATE MODELS OF ISM}

Table 17 Univariate Models of $I S M_{t}$

\begin{tabular}{llllll}
\hline \hline & & & & \\
& $(1)$ & $(2)$ & $(3)$ & $(4)$ & $(5)$ \\
$I S M_{t-1}$ & $\mathrm{AR}(1)$ & $\mathrm{AR}(4)$ & $\operatorname{ARMA}(1,1)$ & $\operatorname{ARMA}(1,2)$ & $\operatorname{ARMA}(4,1)$ \\
\cline { 2 - 6 } & $0.921^{* * *}$ & $0.972^{* * *}$ & $0.909^{* * *}$ & $0.892^{* * *}$ & 0.984 \\
$I S M_{t-2}$ & $(0.023)$ & $(0.065)$ & $(0.028)$ & $(0.037)$ & $(0.556)$ \\
& & 0.059 & & & 0.047 \\
$I S M_{t-3}$ & & $(0.101)$ & & & $(0.543)$ \\
& & 0.016 & & & 0.015 \\
$I S M_{t-4}$ & & $(0.120)$ & & & $(0.128)$ \\
& & $-0.160^{*}$ & & & -0.158 \\
$\epsilon_{t-1}$ & & $(0.078)$ & & $0.115)$ \\
& & & 0.084 & $(0.077)$ & $(0.568)$ \\
$\epsilon_{t-2}$ & & & $(0.073)$ & 0.137 & \\
Constant & $53.045^{* * *}$ & $52.953^{* * *}$ & $53.018^{* * *}$ & $53.018^{* * *}$ & $52.953^{* * *}$ \\
& $(1.818)$ & $(1.285)$ & $(1.696)$ & $(1.596)$ & $(1.283)$ \\
\hline$N$ & 182 & 182 & 182 & 182 & 182 \\
AIC & 746.537 & 742.370 & 747.163 & 746.366 & 744.369 \\
BIC & 756.150 & 761.594 & 759.979 & 762.386 & 766.797 \\
RMSE & 1.841 & 1.789 & 1.834 & 1.820 & 1.789 \\
\hline
\end{tabular}

Note: Standard errors in parentheses; ${ }^{*} p<0.05,{ }^{* *} p<0.01,{ }^{* *} p<0.001$. 


\section{A.3.2 LINEAR MODELS}

Figure 16 Linear models of ISM with Contemporaneous and Lagged Regressors. Observed Values and

\section{Predictions}

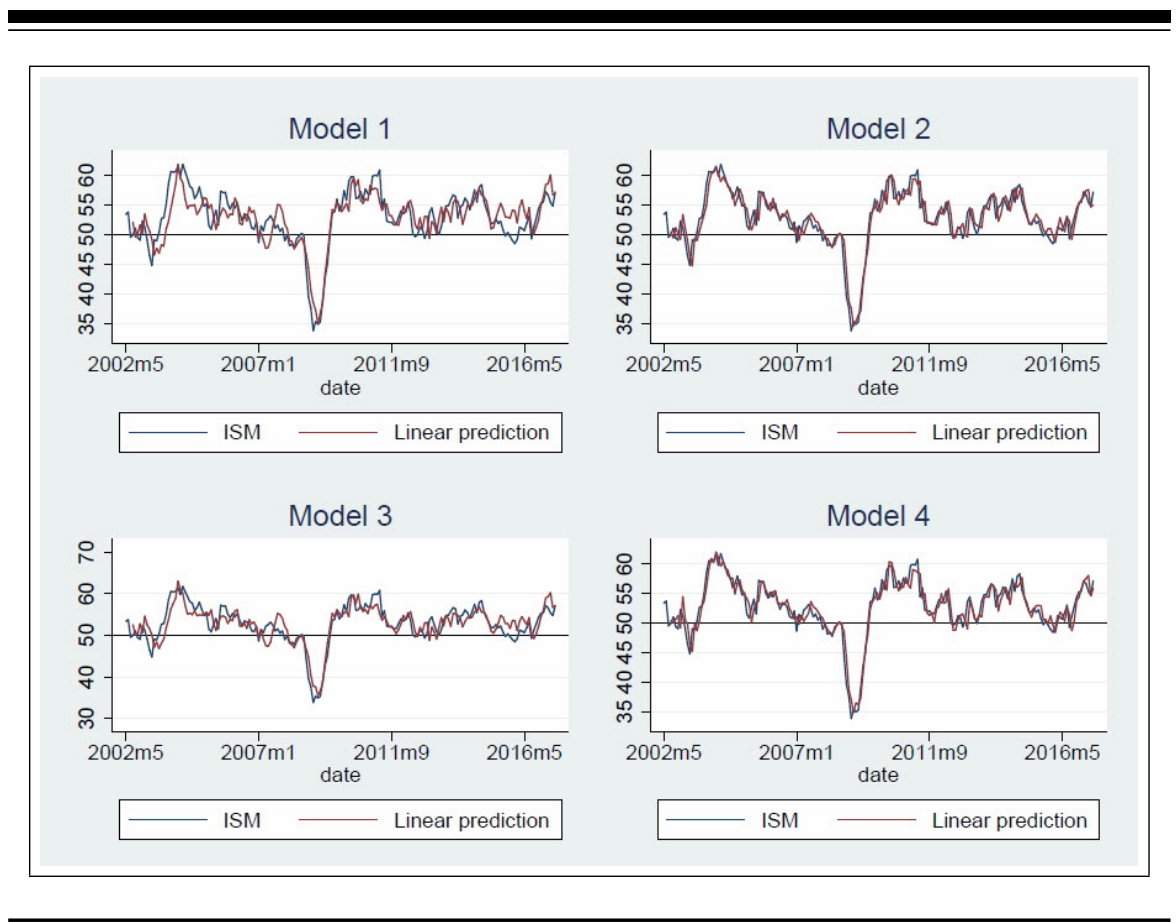




\section{A.3.3 BIVARIATE VAR: ISM AND FRBR} COMPOSITE DIFFUSION INDICES

Table 18 VAR(1): $I S M_{t}$ and $R I C_{t}$. Estimates, Variance-Covariance Matrix, and Cholesky Decomposition

\begin{tabular}{llll}
\hline \hline$\Phi$ & & & \\
& $R I C_{t}$ & $0.35^{* * *}$ & $0.59^{* * *}$ \\
& $I S M_{t}$ & 0.05 & $0.88^{* * *}$ \\
\hline$\alpha$ & $R I C_{t}$ & 1.62 & \\
\hline$\Sigma$ & $I S M_{t}$ & $3.97^{* * *}$ & \\
& $R I C_{t}$ & 12.27 & 3.38 \\
\hline$p-1$ & $I S M_{t}$ & 2.31 & 0.58 \\
\hline$N$ & $R I C_{t}$ & 0.29 & \\
AIC & $I S M_{t}$ & -0.11 & \\
BIC & & 181 & \\
RMSE & & 9.33 & \\
\hline
\end{tabular}


Figure 17 VAR(1): $I S M_{t}$ and $R I C_{t}$. Observed and Predicted Values

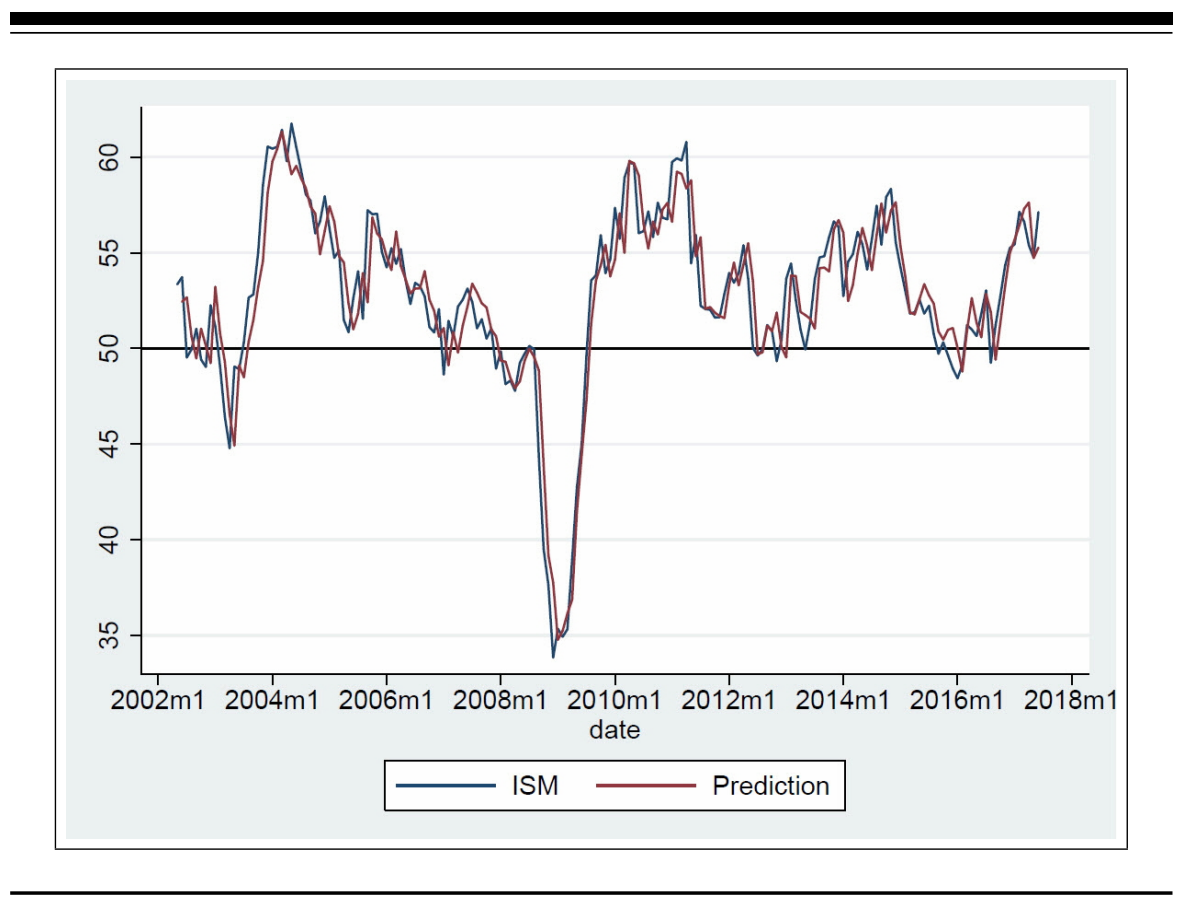




\section{A.3.4 MULTIVARIATE VAR: ISM AND FRBR INDIVIDUAL DIFFUSION INDICES}

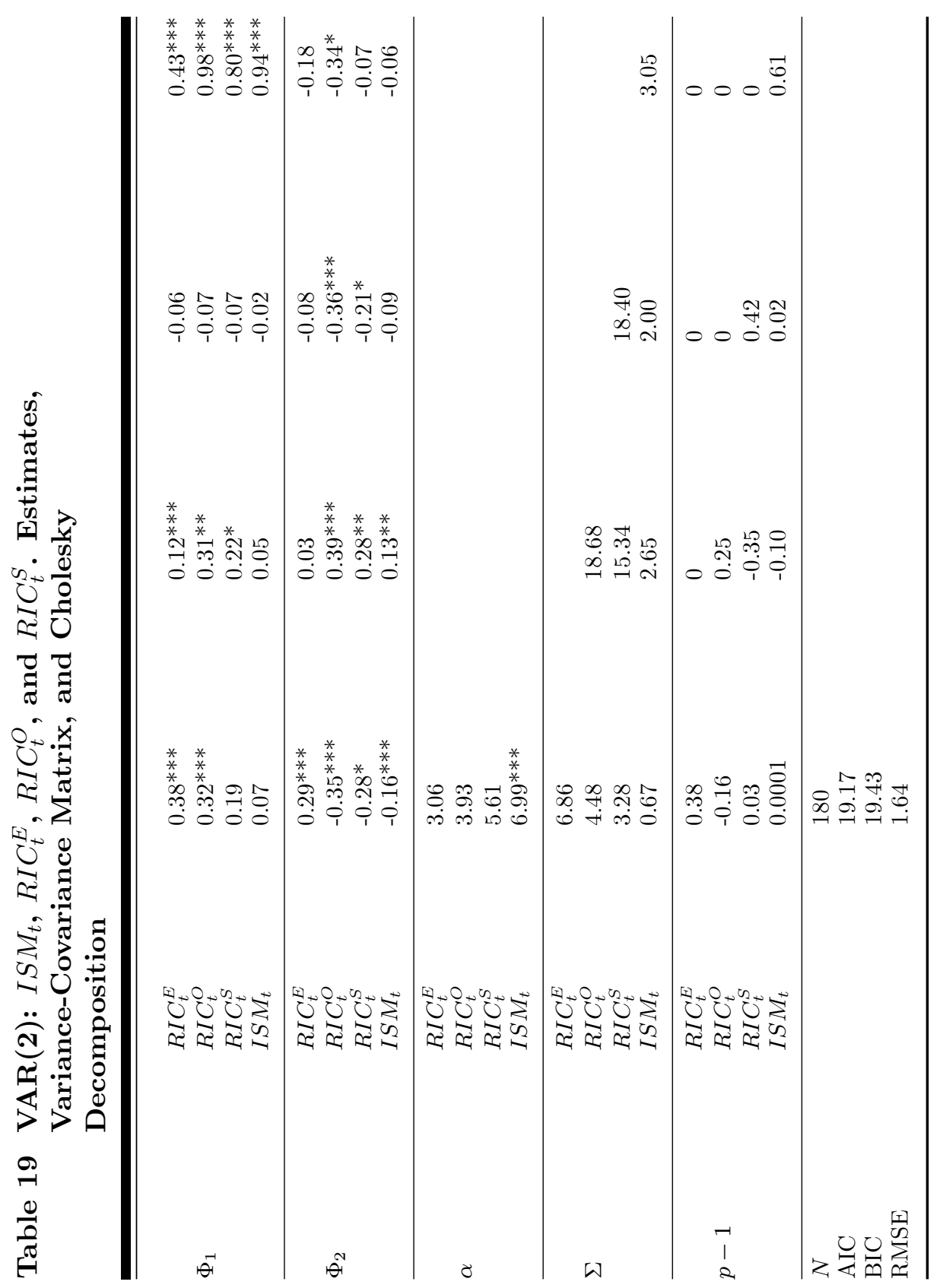


Figure 18 VAR(2): ISM, $R I C_{t}^{E}, R I C_{t}^{O}$, and $R I C_{t}^{S}$. Observed and Predicted Values

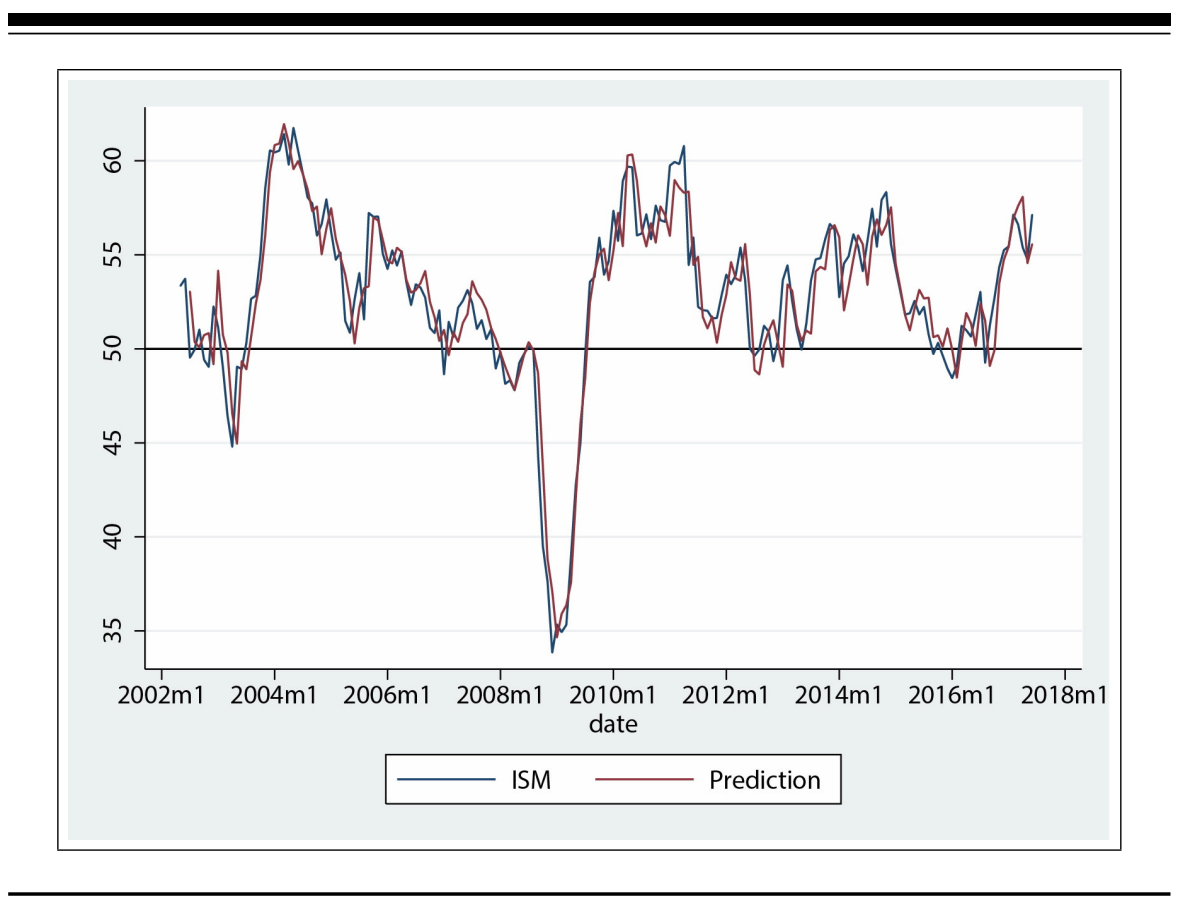




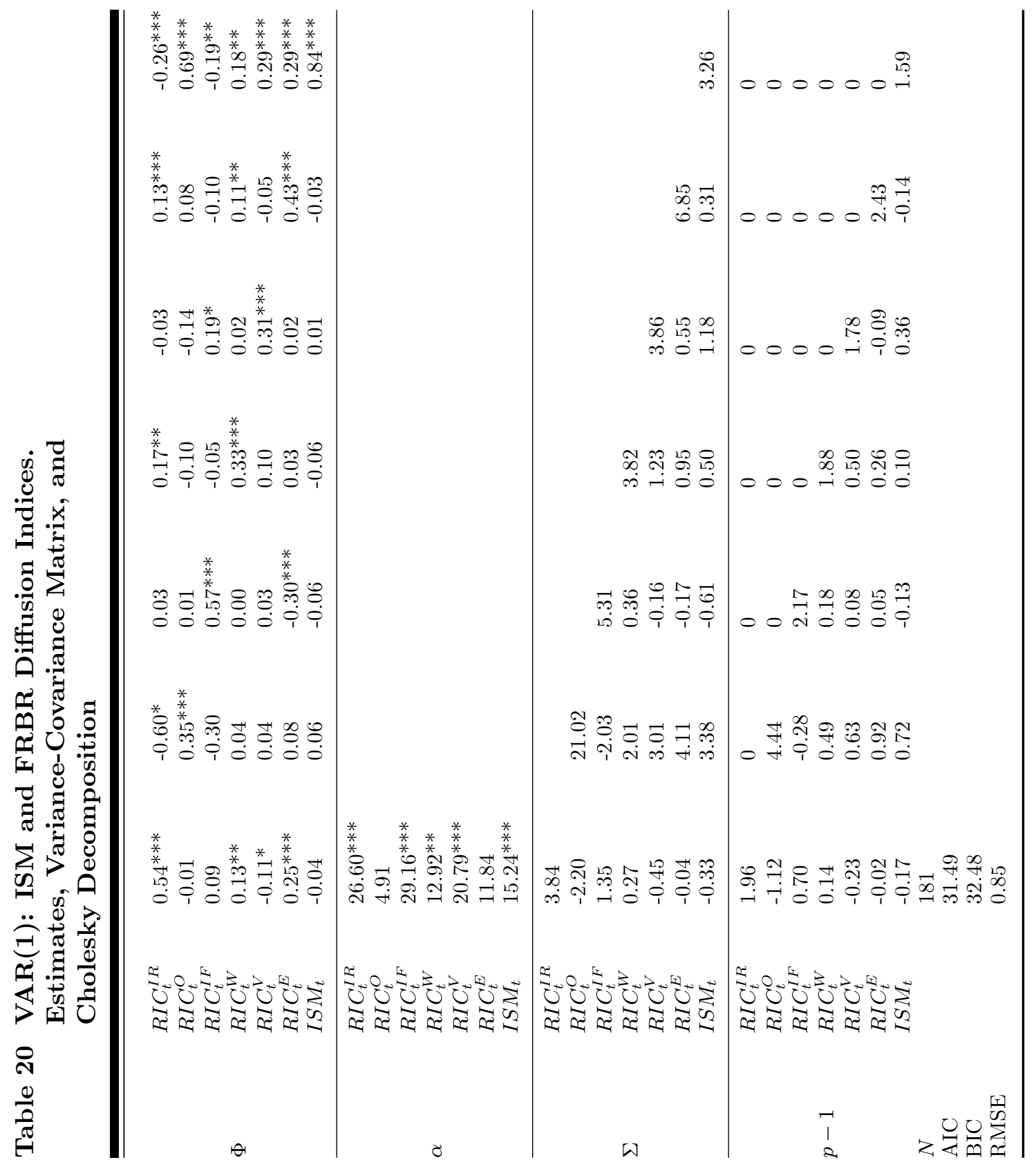


Figure 19 VAR(1): ISM and FRBR Diffusion Indices. Observed and Predicted Values

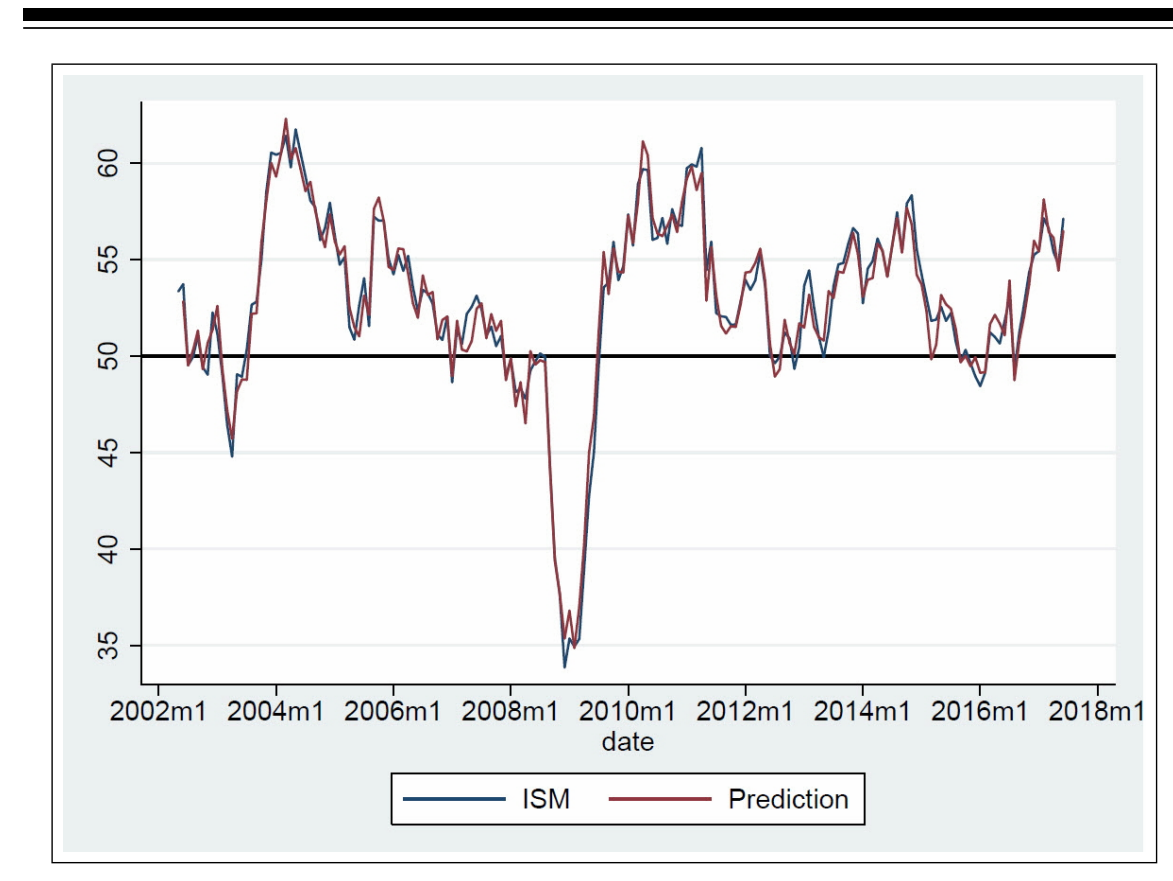




\section{APPENDIX A.4 REGIONAL ECONOMY: VAR MODELS}

\section{A.4.1 BIVARIATE VAR: MEG AND FRBR COMPOSITE INDEX}

Table 21 Bivariate VAR(1): $M E G_{t}$ and $R I C_{t}$. Estimates, Variance-Covariance Matrix, and Cholesky Decomposition

\begin{tabular}{llll}
\hline & & & \\
& $R I C_{t}$ & $0.635^{* * *}$ & $4.851^{* *}$ \\
& $M E G_{t}$ & $0.010^{* * *}$ & $0.596^{* * *}$ \\
\hline \multirow{2}{\alpha}{} & $R I C_{t}$ & 1.081 & \\
\hline & $M E G_{t}$ & $-0.074^{* * *}$ & \\
$\Sigma$ & $R I C_{t}$ & 58.520 & 0.050 \\
\hline & $M E G_{t}$ & 0.448 & 0 \\
$p-1$ & $R I C_{t}$ & 0.131 & 4.619 \\
\hline$N$ & $M E G_{t}$ & -0.035 & \\
AIC & & 180 & \\
BIC & & 6.751 & \\
RMSE & & 6.858 & \\
\hline
\end{tabular}

Figure 20 Bivariate $\operatorname{VAR}(1): M E G_{t}$ and $R I C_{t}$. Observed and Predicted Values

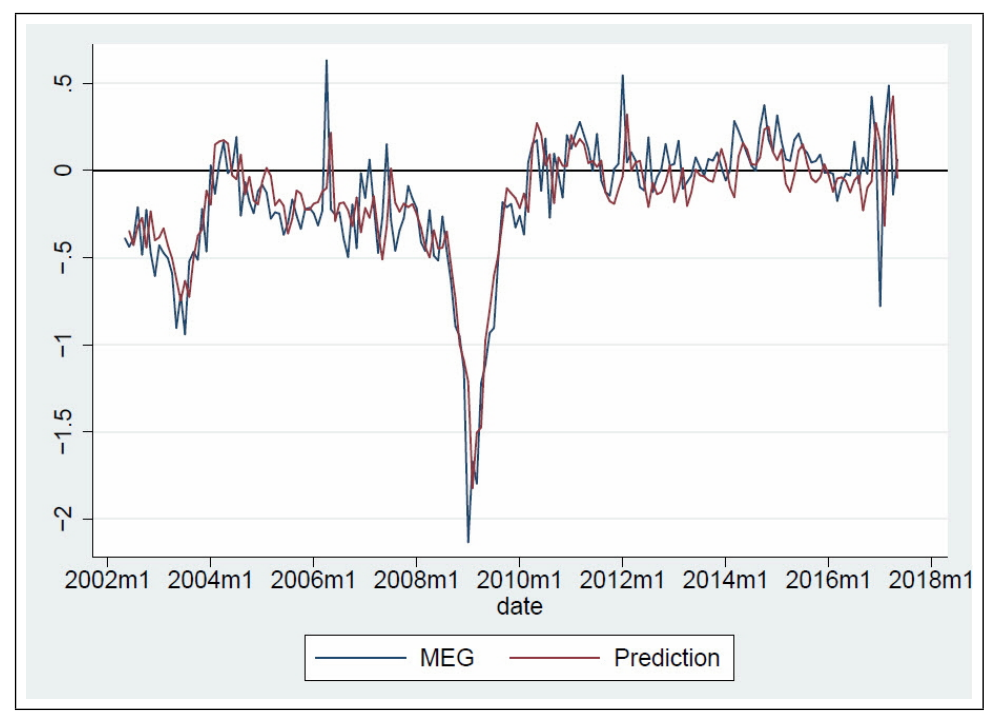


Table 22 Bivariate $\operatorname{VAR}(1): M E G_{t}$ and $R I C_{t}^{E}$. Estimates, Variance-Covariance Matrix, and Cholesky Decomposition

\begin{tabular}{llll}
\hline \hline & & & \\
$\Phi$ & $R I C_{t}^{E}$ & $0.542^{* * *}$ & $8.534^{* * *}$ \\
& $M E G_{t}$ & $0.015^{* * *}$ & $0.490^{* * *}$ \\
\hline \multirow{2}{*}{$R I C_{t}^{E}$} & $1.349^{* *}$ & \\
\hline \multirow{2}{*}{} & $M E G_{t}$ & $-0.079^{* * *}$ & \\
\hline & $R I C_{t}^{E}$ & 32.348 & 0.048 \\
\hline-1 & $M E G_{t}$ & 0.349 & 0 \\
\hline$N$ & $R I C_{t}^{E}$ & 0.176 & 4.761 \\
AIC & $M E G_{t}$ & -0.051 & \\
BIC & & 180 & \\
RMSE & & 6.098 & \\
\hline
\end{tabular}

Figure 21 Bivariate $\operatorname{VAR}(1): M E G_{t}$ and $R I C_{t}^{E}$. Observed and Predicted Values

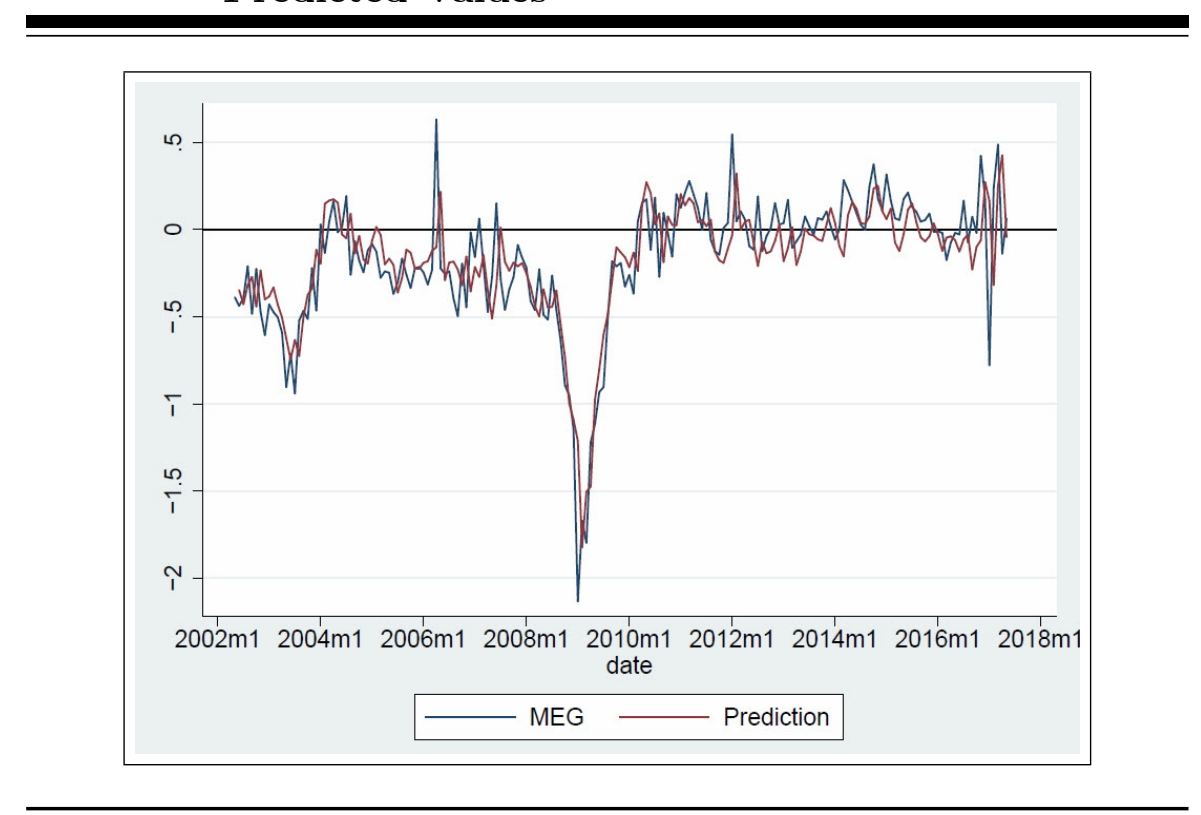




\section{A.4.2 MULTIVARIATE VAR MODEL: MEG AND FRBR INDIVIDUAL DIFFUSION INDICES}

Figure 22 VAR(1) model: MEG and FRBR Individual Diffusion Indices. Observed and Predicted Values

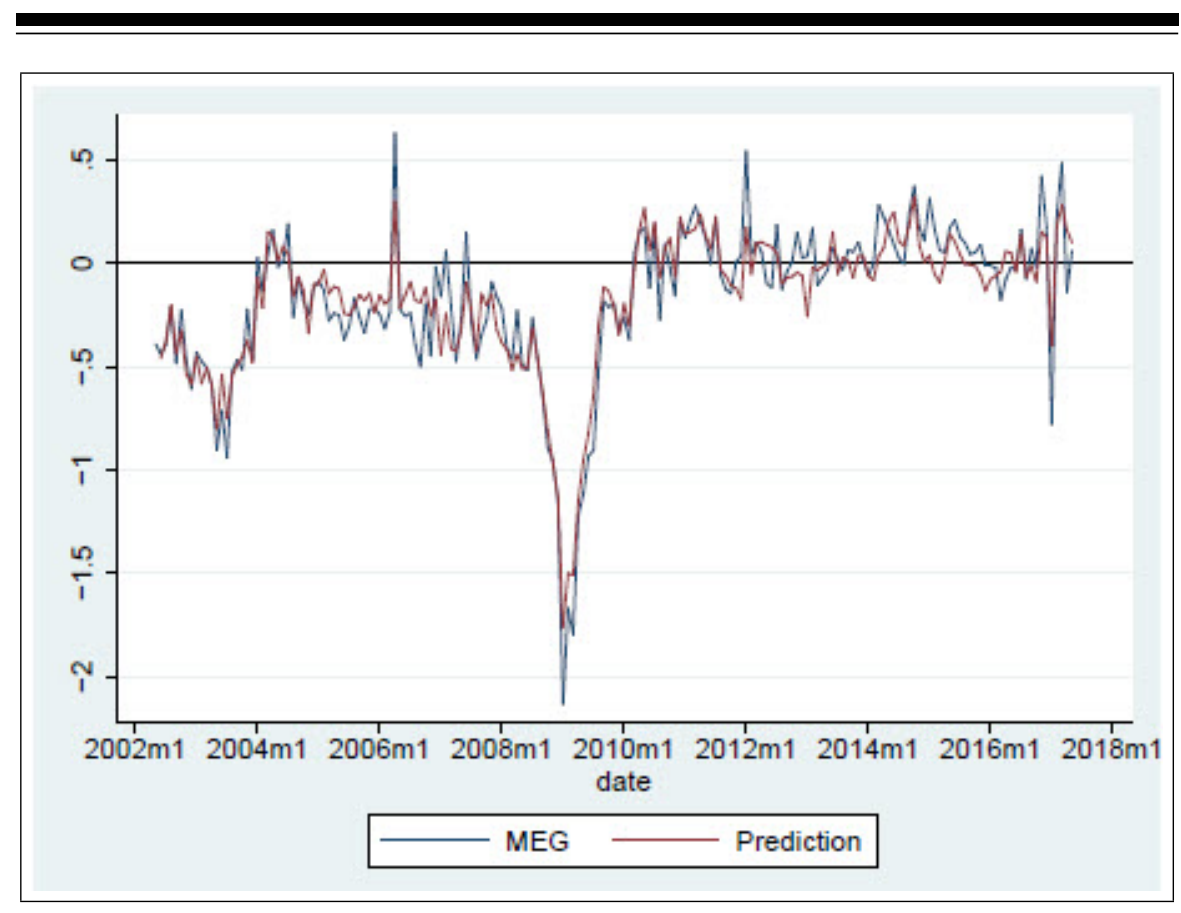




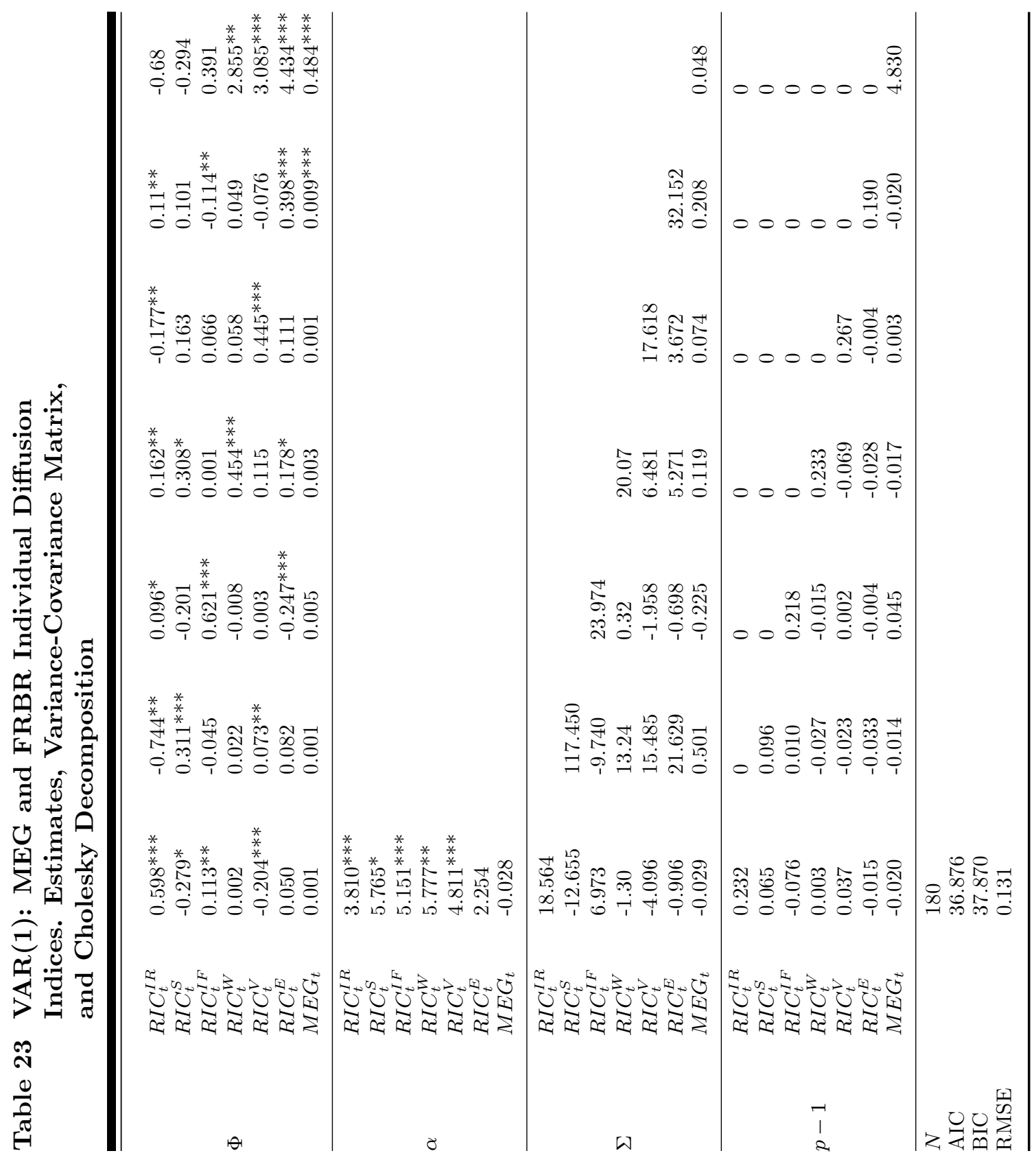




\section{REFERENCES}

Harris, Matthew, Raymond E. Owens, and Pierre-Daniel G. Sarte. 2004. "Using Manufacturing Surveys to Assess Economic Conditions." Federal Reserve Bank of Richmond Economic Quarterly 90 (Fall): 65-93.

Pinto, Santiago, Pierre-Daniel G. Sarte, and Robert Sharp. 2015a. "Learning About Consumer Uncertainty from Qualitative Surveys: As Uncertain As Ever." Federal Reserve Bank of Richmond Working Paper 15-09 (August).

Pinto, Santiago, Sonya Ravindranath Waddell, and Pierre-Daniel G. Sarte. 2015b. "Monitoring Economic Activity in Real Time Using Diffusion Indices: Evidence from the Fifth District." Federal Reserve Bank of Richmond Economic Quarterly 101 (Fourth Quarter): 275-301. 
